\title{
Bone marrow adipogenic lineage precursors promote osteoclastogenesis in bone remodeling and pathologic bone loss
}

\author{
Wei Yu, ${ }^{1,2}$ Leilei Zhong, ${ }^{1}$ Lutian Yao, ${ }^{1}$ Yulong Wei, ${ }^{1,2}$ Tao Gui, ${ }^{1,3}$ Ziqing Li, ${ }^{4}$ Hyunsoo Kim, ${ }^{5}$ Nicholas Holdreith, ${ }^{6,7}$ Xi Jiang, ${ }^{1}$ \\ Wei Tong, ${ }^{6,7}$ Nathaniel Dyment, ${ }^{1} X$. Sherry Liu, ${ }^{1}$ Shuying Yang, ${ }^{4}$ Yongwon Choi, ${ }^{5}$ Jaimo Ahn, ${ }^{1}$ and Ling Qin ${ }^{1}$ \\ 'Department of Orthopaedic Surgery, Perelman School of Medicine, University of Pennsylvania, Philadelphia, Pennsylvania, USA. ${ }^{2}$ Department of Orthopaedics, Union Hospital, Tongji Medical College, \\ Huazhong University of Science and Technology, Wuhan, China. ${ }^{3}$ Department of Bone and Joint Surgery, Institute of Orthopedic Diseases, The First Affiliated Hospital, Jinan University, Guangzhou, \\ Guangdong, China. ${ }^{4}$ Department of Basic \& Translational Sciences, School of Dental Medicine, University of Pennsylvania, Philadelphia, Pennsylvania, USA. ${ }^{5}$ Department of Pathology and Laboratory \\ Medicine, Perelman School of Medicine, University of Pennsylvania, Philadelphia, Pennsylvania, USA. ${ }^{6}$ Division of Hematology, Children's Hospital of Philadelphia, Philadelphia, Pennsylvania, USA. \\ ${ }^{7}$ Department of Pediatrics, Perelman School of Medicine at the University of Pennsylvania, Philadelphia, Pennsylvania, USA.
}

Bone is maintained by coupled activities of bone-forming osteoblasts/osteocytes and bone-resorbing osteoclasts. Alterations in this relationship can lead to pathologic bone loss such as osteoporosis. It is well known that osteogenic cells support osteoclastogenesis via production of RANKL. Interestingly, our recently identified bone marrow mesenchymal cell population-marrow adipogenic lineage precursors (MALPs) that form a multidimensional cell network in bone-was computationally demonstrated to be the most interactive with monocyte-macrophage lineage cells through high and specific expression of several osteoclast regulatory factors, including RANKL. Using an adipocyte-specific Adipoq-Cre to label MALPs, we demonstrated that mice with RANKL deficiency in MALPs have a drastic increase in trabecular bone mass in long bones and vertebrae starting from 1 month of age, while their cortical bone appears normal. This phenotype was accompanied by diminished osteoclast number and attenuated bone formation at the trabecular bone surface. Reduced RANKL signaling in calvarial MALPs abolished osteolytic lesions after LPS injections. Furthermore, in ovariectomized mice, elevated bone resorption was partially attenuated by RANKL deficiency in MALPs. In summary, our studies identified MALPs as a critical player in controlling bone remodeling during normal bone metabolism and pathological bone loss in a RANKL-dependent fashion.

\section{Introduction}

Bone is a dynamic tissue, constantly undergoing remodeling through coupled activities of bone-resorbing osteoclasts and bone-forming osteoblasts/osteocytes. A shift in the balance of these 2 actions toward resorption leads to osteoporosis, an insidious disease characterized by excessive bone loss, microarchitectural deterioration, and increased risk of fracture. As a highly prevalent disorder, osteoporosis affects more than 75 million people in the United States, Europe, and Japan and is the underlying condition related to more than 8.9 million fractures annually worldwide (1).

Mature osteoclasts are large multinucleated cells derived from the monocyte-macrophage lineage of hematopoietic origin (2). They firmly attach to the bone surface and degrade bone matrix. Osteoclast differentiation predominantly depends on RANKL signaling (encoded by Tnfsf11 gene), a type II transmembrane protein of the TNF superfamily, and is modulated by other cytokines and

Conflict of interest: The authors have declared that no conflict of interest exists. Copyright: () 2021, American Society for Clinical Investigation.

Submitted: May 13, 2020; Accepted: October 29, 2020; Published: January 19, 2021.

Reference information: J Clin Invest. 2021;131(2):e140214.

https://doi.org/10.1172/JCl140214. growth factors (3). Tnfsf $11^{-/-}$mice have no osteoclasts in bone and exhibit a severe osteopetrosis (high bone mass) phenotype $(4,5)$. Early studies indicated that osteoblasts and their progenitors are the major source of RANKL in bone to support osteoclastogenesis (6). Later, animal studies showed that osteoblast ablation does not affect osteoclast formation $(7,8)$. In growing mice, hypertrophic chondrocytes appear to be the main source of RANKL for bone resorption (9). In adult mice, osteocytes, the descendants of osteoblasts that are embedded in the bone matrix, have been demonstrated to be the major stimulator of osteoclastogenesis (9-11).

Osteoblasts and osteocytes are derived from bone marrow mesenchymal stem cells (MSCs), which also give rise to marrow adipocytes. Recently, we computationally delineated the hierarchy of mesenchymal lineage cells from MSCs to mature cells using large-scale, single-cell RNA-sequencing (scRNA-seq). Surprisingly, this study unveiled a new cell population, marrow adipogenic lineage precursors (MALPs), situating along the adipogenic differentiation route after mesenchymal progenitors and before classic lipid-laden adipocytes (LiLAs) (12). Labeled by mature adipocyte-specific Adipoq-Cre (13), MALPs are abundant, nonproliferative cells that express many adipocyte markers but have no lipid accumulation. Shaped as a central body with multiple cell processes, they exist as stromal cells and pericytes forming a 3D network 
ubiquitously distributed within the bone marrow, and function to maintain vessel structure and inhibit bone formation.

Our scRNA-seq data sets were derived from analyzing $\mathrm{Td}^{+}$ cells sorted from endosteal bone marrow of Col2-Cre RosatdTomato (Col2/Td) mice at various ages. In these mice, Td labels the entire mesenchymal lineage $(12,14,15)$. Interestingly, our data sets also contained many hematopoietic cells, including osteoclasts. While these contaminant cells did not affect the single-cell analysis of mesenchymal lineage cells, they actually provided an advantage for delineating the osteoclast differentiation process and for examining the interaction between osteoclastogenesis and mesenchymal subpopulations. To our surprise, our in silico data indicated that MALPs, not osteoblasts or osteocytes, are the most supportive cells for osteoclast formation. To validate this finding, we constructed adipocyte-specific RANKL-CKO mice to investigate the role of MALP-derived RANKL in regulating bone remodeling at various skeletal sites under physiological and pathological conditions.

\section{Results}

scRNA-seq analysis reveals osteoclastogenesis in bone marrow. To identify bone marrow mesenchymal subpopulations and to delineate the bilineage differentiation paths of bone marrow MSCs, we performed scRNA-seq on top $1 \% \mathrm{Td}^{+}$cells sorted from endosteal bone marrow of 1- to 3-month-old Col2/Td mice. Combining 3 batches of sequencing data generated a data set containing 17,494 cells with 2519 genes/cell and 11,071 unique molecular identifiers (UMIs) per cell. Clustering analysis revealed 20 cell clusters (Figure 1A), including 8 mesenchymal lineage cell clusters (markers for 6 clusters are shown in Supplemental Figure 1A; supplemental material available online with this article; https://doi.org/10.1172/ JCI140214DS1), 10 hematopoietic lineage cell clusters, 1 endothelial cell cluster, and 1 mural cell cluster. Our previous study annotated mesenchymal clusters as early mesenchymal progenitors (EMPs), intermediate mesenchymal progenitors (IMPs), late mesenchymal progenitors (LMPs), lineage committed progenitors (LCPs), osteoblasts, osteocytes, MALPs, and chondrocytes (12).

Hematopoietic cells were commonly observed in recently published scRNA-seq data sets of bone marrow mesenchymal lineage cells (16-19). In our mouse model, it appears that hematopoietic cells do express the Td gene albeit at a much lower level than mesenchymal cells (Supplemental Figure 1B). Flow analysis of bone marrow cells confirmed that all hematopoietic subpopulations, ranging from progenitors to mature cells, contain a subset of $\mathrm{Td}^{+}$ cells whose relative abundance in the bone marrow correlated well to the cell populations captured in scRNA-seq analysis (Supplemental Table 1). According to known lineage markers, hematopoietic cells in the sequencing data set were divided into hematopoietic stem and progenitor cells (HSPCs), monocyte progenitors (MP), granulocyte progenitors (GrP), granulocytes, B cells, T cells, erythrocytes (Supplemental Figure $1 \mathrm{C}$ ), monocytes, macrophages, and osteoclasts (Figure 1B). Hierarchy analysis showed distinct gene expression signature in each cluster (Supplemental Figure 1D).

Osteoclasts in postnatal mice are mostly derived from monocyte-macrophage lineage of HSPC descendants. Indeed, monocyte, macrophage, and osteoclast cells (clusters 13, 14, and 15, respectively) were close to each other in the uniform manifold approximation and projection (UMAP) plot. Separately analyzing these cells using UMAP or Monocle generated one monocyte cluster at one end of the pseudotime trajectory, one macrophage cluster $(\mathrm{M} \phi \alpha)$ at the branch point, one macrophage cluster $(\mathrm{M} \phi \beta)$ at the second end, and one early osteoclast cluster and one late osteoclast cluster at the third end (Figure 1, C and D), suggesting that monocytes undergo bilineage differentiation into mature $\mathrm{M} \phi \beta$ cells and osteoclasts with $\mathrm{M} \phi \alpha$ as the intermediate cell type. Genes related to mature osteoclast functions, such as fusion, matrix digestion, and proton translocation, were highly expressed in the late osteoclast cluster (Figure 1E). Cell-cycle analysis confirmed that terminal differentiated $\mathrm{M} \phi \beta$ cells and late osteoclasts are nonproliferative whereas other cells, particularly early osteoclasts, are highly proliferative (Figure 1F).

Positioning individual cells along a linear pseudo-timeline with monocytes as the root revealed known and novel transcription factors (TFs) differentially expressed after the branch point into 2 lineages (Figure 1G). Nfatc1, a master regulator of osteoclast differentiation (20), was present within the osteoclast lineage differentiation, supporting the reliability of our computational analysis. Other known TFs, such as Ppargc1b (21), Mitf (22), Ezh2 (23), and Hmgb2 (24), were also identified in this assay. To date, TFs driving macrophage differentiation inside the bone marrow are largely unknown. Our analysis suggested that some adipogenic TFs, such as Pparg, Cebpa, and Cebpb, and Notch signaling TFs, such as Notch 2 and Hes1, are upregulated during the differentiation route of macrophages in bone. These results are consistent with previous studies demonstrating a similar upregulation of these TFs during either in vitro macrophage activation assay or macrophage differentiation in nonosseus tissues from blood monocytes (25-28).

Gene Ontology (GO) term and Kyoto Encyclopedia of Genes and Genomes (KEGG) pathway analyses of differentially expressed genes (DEGs) between early and late osteoclasts pointed out many known features of osteoclasts, such as proton transport, ion transport, ATP biogenesis, and mitochondrial related metabolic pathways (Figure $1 \mathrm{H}$ and Supplemental Table 2). Early osteoclasts were enriched with cell-cycle genes, indicating their proliferative nature (Supplemental Figure 2A). Comparison of 2 macrophage clusters indicated that the main function of $M \phi \beta$ is efferocytosis because pathways such as apoptotic cell clearance, endocytosis, oxidation-reduction processes, and lipid metabolic were enriched in this cluster of cells (Figure 1I and Supplemental Table 3). In contrast, $\mathrm{M} \phi \alpha$ was associated with translation, immune-response, and chemotaxis (Supplemental Figure 2B), suggesting that these cells are more involved in regulating their environment. Collectively, our scRNA-seq data set provided a powerful tool for analyzing the in vivo differentiation route of osteoclasts as well as cellular functional changes.

MALPs are specifically labeled by Adipoq-Cre in adult mice. Our previous study used mature adipocyte-specific Adipoq-Cre to label MALPs in 1-month-old mice. Since marker gene expression could be fluid among mesenchymal subpopulations during aging (12), we first investigated whether the same specificity holds in adult mice. For this purpose, we constructed Adipoq-Cre Tomato (Adipoq/Td) mice with or without 2.3kbCol1a1-GFP (Col1-GFP) that labels osteoblasts (29). At 3 months of age, Adipoq/Td/Col1GFP mice showed prominent Td signal inside the bone marrow 
A
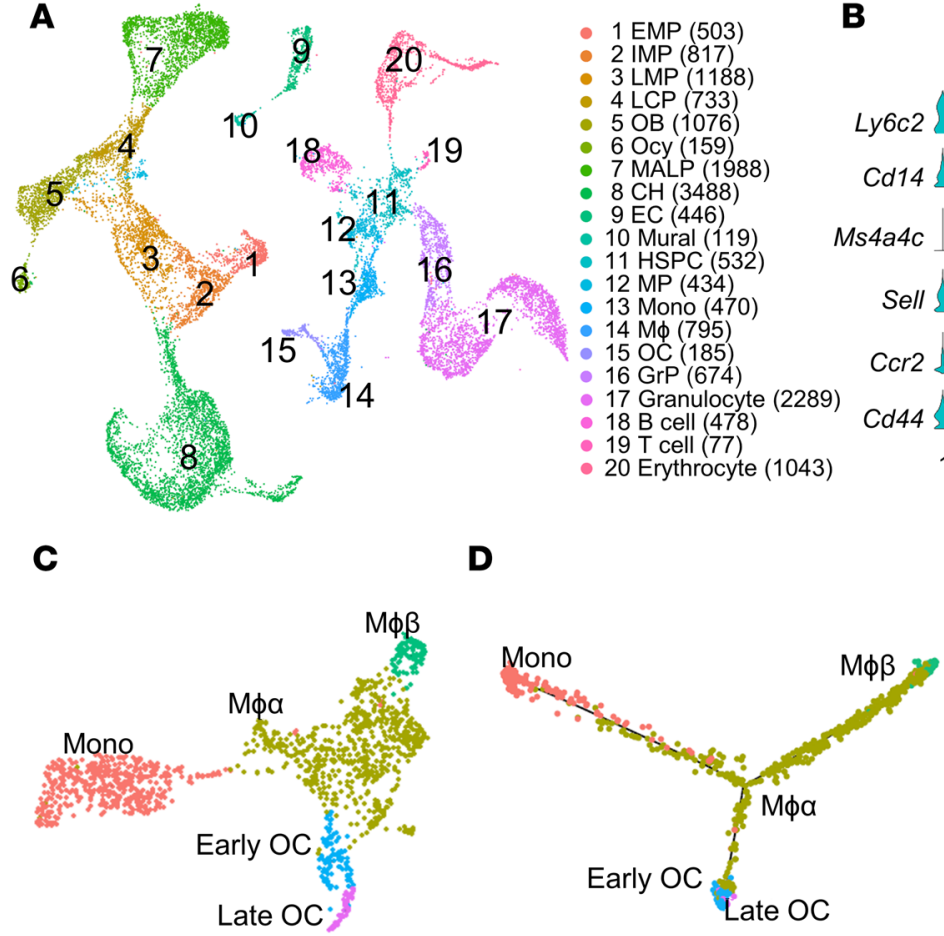

G
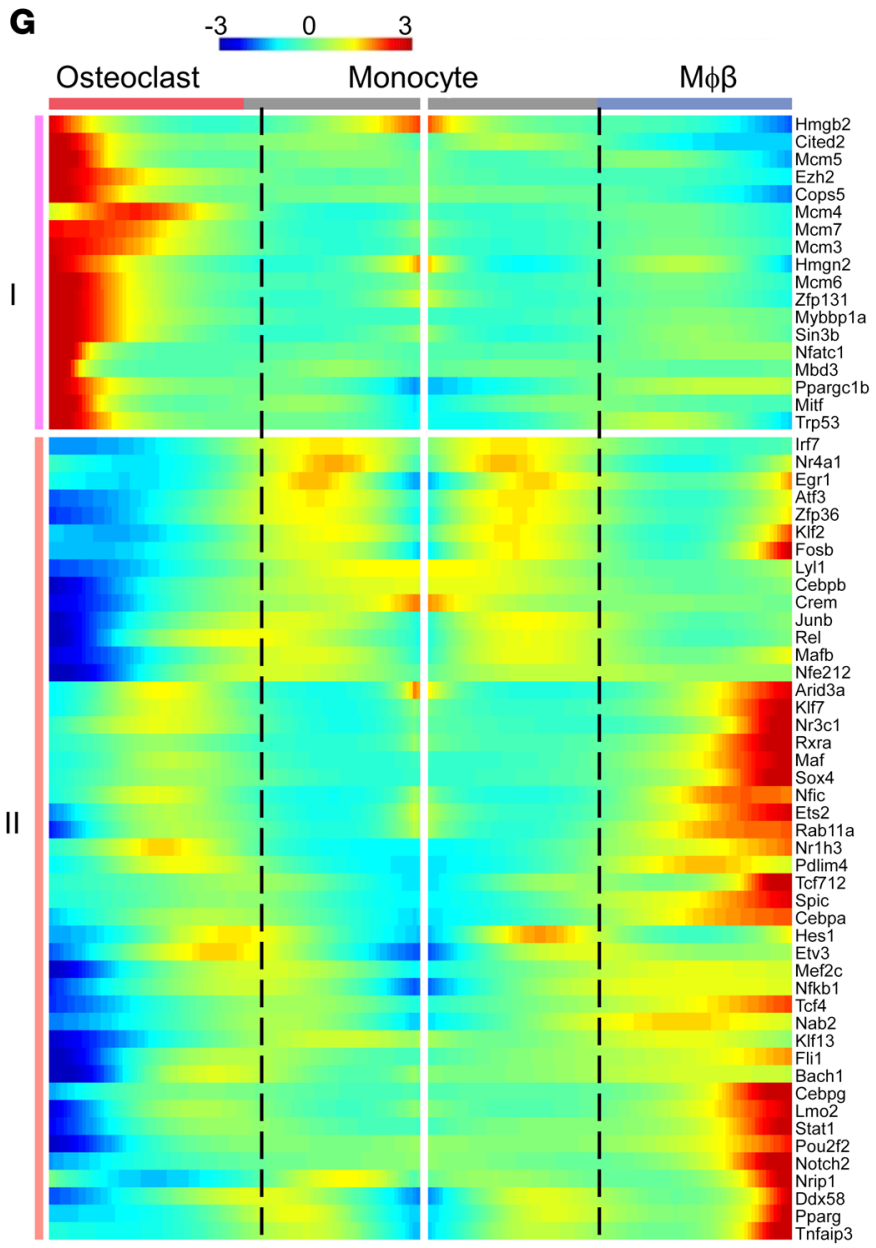
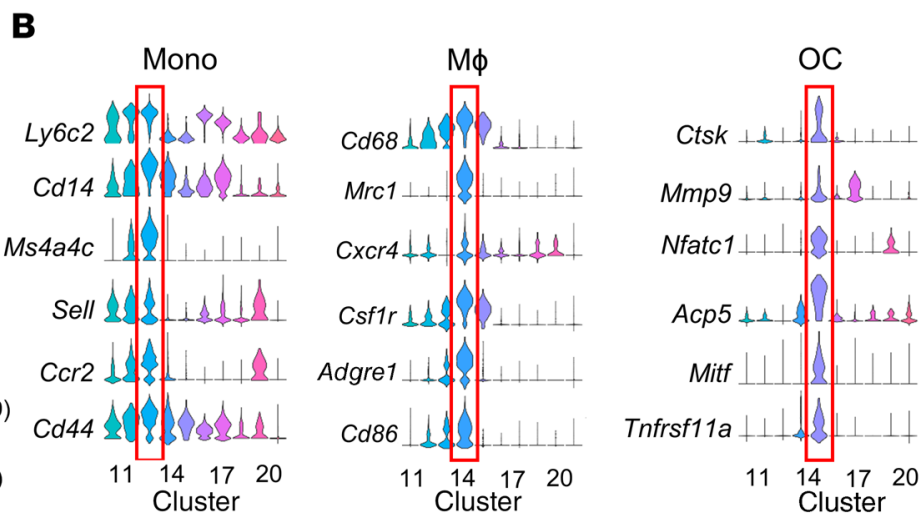

E

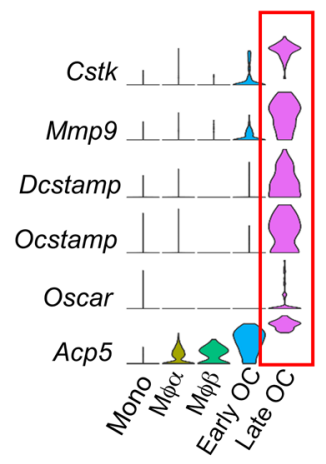

$\mathbf{F}$

Proliferative cells

(\%)

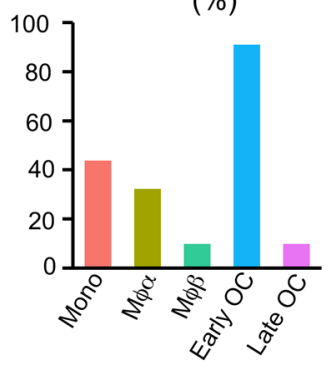

H

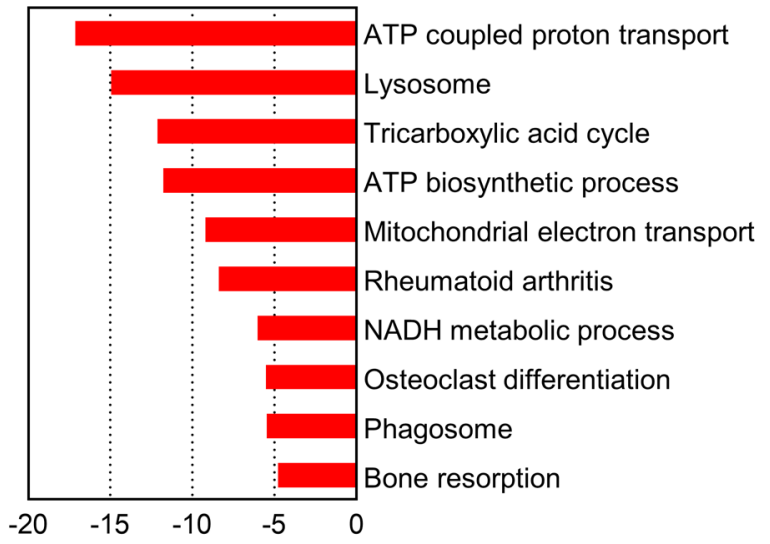

I

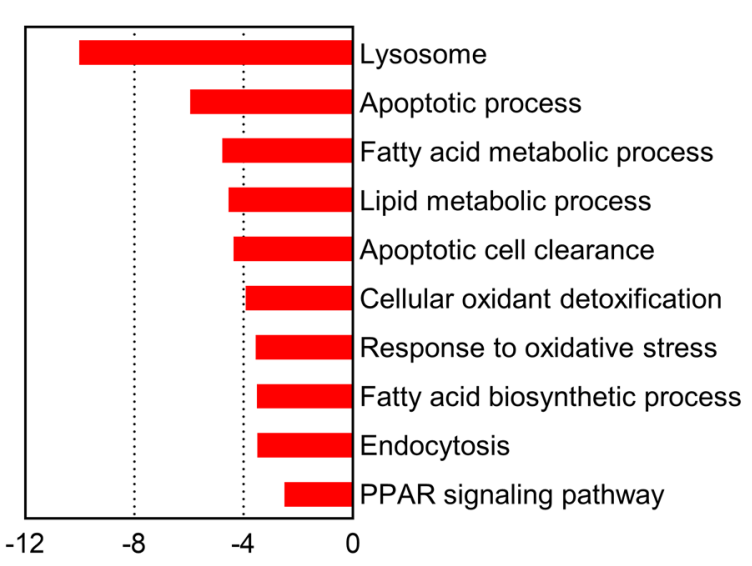


Figure 1. Single-cell RNA sequencing identifies bone marrow monocyte-macrophage lineage cells and delineates in vivo osteoclastogenesis. (A) The UMAP plot of cells isolated from bone marrow of 1- to 3-month-old Col2/Td mice ( $n=8$ mice). Ocy, osteocyte; $\mathrm{CH}$, chondrocyte; EC, endothelial cells; MP, monocyte progenitor; M $\phi$, macrophage; OC, osteoclast; GrP, granulocyte progenitor. (B) Violin plots of marker gene expression for monocyte, macrophage, and osteoclast clusters. (C) The UMAP plot of monocyte-macrophage lineage cells only. (D) Monocle trajectory plot of monocyte-macrophage lineage cells. (E) Violin plots of known late osteoclast markers. (F) The percentage of proliferative cells (S/G2/M phase) among each cluster was quantified. (G) Pseudotemporal depiction of differentially expressed TFs starting from the branch point (dashed lines) toward osteoclast (left) and macrophage (right) differentiation. Groups I and II contain TFs that are highly upregulated during osteoclast and macrophage differentiation routes, respectively. Color bar indicates the gene expression level. (H) GO term and KEGG pathway analyses of genes upregulated in late osteoclasts compared with early osteoclasts. (I) GO term and KEGG pathway analyses of genes upregulated in M $\phi \beta$ cells compared with M $\phi \alpha$ cells.

of long bones (Figure 2A). However, chondrocytes in articular and growth plate cartilage were not labeled (Figure 2, B and C). $\mathrm{Td}^{+}$cells were present at the chondro-osseous junction (COJ) between growth plate and primary spongiosa and throughout the entire metaphyseal and diaphyseal bone marrow. Very few osteoblasts (defined as $\mathrm{GFP}^{+}$bone surface cells) and osteocytes (defined as bone matrix embedded cells) were $\mathrm{Td}^{+}$(Figure 2, D, E, and G), indicating that Adipoq-Cre rarely labels bone-forming cells in adult mice. We did observe some $\mathrm{Td}^{+}$cells at the bone surface but they were often GFP- (Figure 2D). Furthermore, we did not find any $\mathrm{Td}^{+}$cells at the periosteal surface of cortical bone (Figure $2 \mathrm{~F}$ ). Inside the bone marrow, $\mathrm{Td}^{+}$cells existed as pericytes surrounding capillaries (Figure $2 \mathrm{H}$ ) and nonhematopoietic stromal cells (Figure 2I). They did not incorporate EdU (Figure 2J), suggesting a nonproliferative nature. As expected, all Perilipin ${ }^{+}$ bone marrow adipocytes were $\mathrm{Td}^{+}$(Figure $2 \mathrm{~K}$ ).

To further examine whether Adipoq-Cre labels progenitors, we cultured bone marrow mesenchymal progenitors for osteogenic and adipogenic differentiation. As shown in Figure 2L, most CFU-F colonies were made of $100 \% \mathrm{Td}^{-}$cells. A few colonies contained some $\mathrm{Td}^{+}$cells but the majority of cells inside the colony were $\mathrm{Td}^{-}$, indicating that $\mathrm{Td}^{+}$cells lack colony-formation ability and therefore are not proliferative progenitors. When subjected to adipogenic differentiation, $\mathrm{Td}^{-}$progenitors became $\mathrm{Td}^{+}$cells first (day 1-2) and then accumulated lipid droplets (day 4-5) (Figure $2 \mathrm{M}$ ). Notably, all lipid-laden adipocytes were $\mathrm{Td}^{+}$cells. On the contrary, $\mathrm{Td}^{-}$progenitors started to form bony nodules around day 8-10 and maintained as $\mathrm{Td}^{-}$cells during osteogenic differentiation process (Figure $2 \mathrm{~N}$ ). Meanwhile, we observed that ascorbic acid in the osteogenic differentiation medium has the ability to modestly increase Adipoq and Pparg, but not Cebpo, expression (Supplemental Figure 3A), resulting in a small percentage of $\mathrm{Td}^{+}$cells in a subset of bony nodules (Supplemental Figure 3B). However, we never found a nodule entirely made of $\mathrm{Td}^{+}$cells. Our data showed that there were 180 times more Perilipin- ${ }^{-} d^{+}$cells (MALPs) than Perilipin ${ }^{+} \mathrm{Td}^{+}$cells (LiLAs) in bone marrow at 3 months of age (3600 MALPs vs. 20 LiLAs of $3620 \mathrm{Td}^{+}$cells counted; $n=3$ mice), demonstrating that Adipoq-Cre is suitable for specific targeting of
MALPs, a nonproliferative, committed adipogenic precursor population in the bone marrow of adult mice.

MALPs are the major source of osteoclast regulatory factors. It is well accepted that mesenchymal lineage cells promote osteoclast precursors to differentiate into mature osteoclasts. With the identification of mesenchymal subpopulations in bone, we next sought to find out which one communicates the most with monocyte-macrophage lineage cells. To do so, we calculated the number of ligand-receptor pairs between each mesenchymal subpopulation and monocytic subpopulation in our scRNA-seq data set. Interestingly, MALPs displayed the most interactions with all 3 monocytic subpopulations, followed by EMPs (Figure 3A). Surprisingly, osteoblasts and osteocytes had the least number of interactions. Within monocytic cells, macrophages had the most interactions with MALPs, followed by osteoclasts.

Among the identified ligand-receptor pairs, the most prominent ones are RANKL-RANK and Csf1-Csf1r, 2 major signals for osteoclastogenesis. Violin plots clearly showed that MALPs are the major source of Tnfsf11 and Csfl among mesenchymal cells (Figure 3B). Other factors known for regulating osteoclast proliferation, migration, and differentiation, such as Il7 (30), Il34 (31), Ccl2 (32), Vcam1 (33), and C3 (34), were also highly expressed in MALPs but not in osteoblasts and osteocytes. The expression of their corresponding receptors was confirmed in monocytic cells (Figure 3C).

Cell-cell interaction is the major mechanism by which RANKL stimulates osteoclast maturation $(35,36)$. In the bone marrow of Adipoq/Td/Coll-GFP mice, we observed that $\mathrm{TRAP}^{+}$, boneattaching osteoclasts are often contacted by cell processes of neighboring $\mathrm{Td}^{+}$MALPs (Figure 3, D-F). In contrast, the direct contact between osteoclasts and $\mathrm{GFP}^{+}$osteoblasts was less frequent. Instead, we often observed that a line of $\mathrm{GFP}^{+}$osteoblasts and a line of $\mathrm{TRAP}^{+}$osteoclasts were located at the opposite sides of a trabecula (Figure 3F). These data indicate that MALPs are more likely to spatially regulate osteoclastogenesis than osteoblasts via RANKL surface expression.

RANKL from MALPs is critical for bone resorption. To study the role of RANKL in adipogenic lineage cells, we first analyzed its expression in the bone marrow of Adipoq/Td mice. Quantitative real-time PCR (qRT-PCR) revealed that $\mathrm{Td}^{+}$cells, which are only $0.74 \%$ of total bone marrow cells as analyzed by flow cytometry, express 15 times more Tnfsf 11 cells than $\mathrm{Td}^{-}$cells (Figure 4A), indicating that MALPs are one of predominant RANKL sources in the bone marrow. Next, we constructed Adipoq-Cre Tnfsf1 $11^{f l f l}$ (RANKL-CKO ${ }^{\text {Adipoq }}$ ) mice. Compared with WT siblings, these mice had 60\% and 75\% decreases in Tnfsf 11 mRNA in bone marrow at 1 and 3 months of age, respectively (Figure $4 \mathrm{~B}$ ). Tnfsf 11 mRNA in cortical bone, however, was unchanged (Figure $4 \mathrm{C}$ ), suggesting that these mice have RANKL deficiency specifically in adipogenic lineage cells within the marrow but not in osteocytes within the cortical bone.

$R A N K L-C K O^{\text {Adipoq }}$ mice displayed normal postnatal growth with unchanged body and spleen weight up to 12 weeks of age (Supplemental Figure 4). Their tooth eruption (Figure 4D) and growth plates (Figure 4, E and F) appeared normal with unaffected long bone growth (Figure 4G). At 1 month of age, male RANKL-CKO ${ }^{\text {Adipoq }}$ mice showed a marked $61 \%$ increase in tibial trabecular bone mass (BV/TV), accompanied by a $63 \%$ increase in 

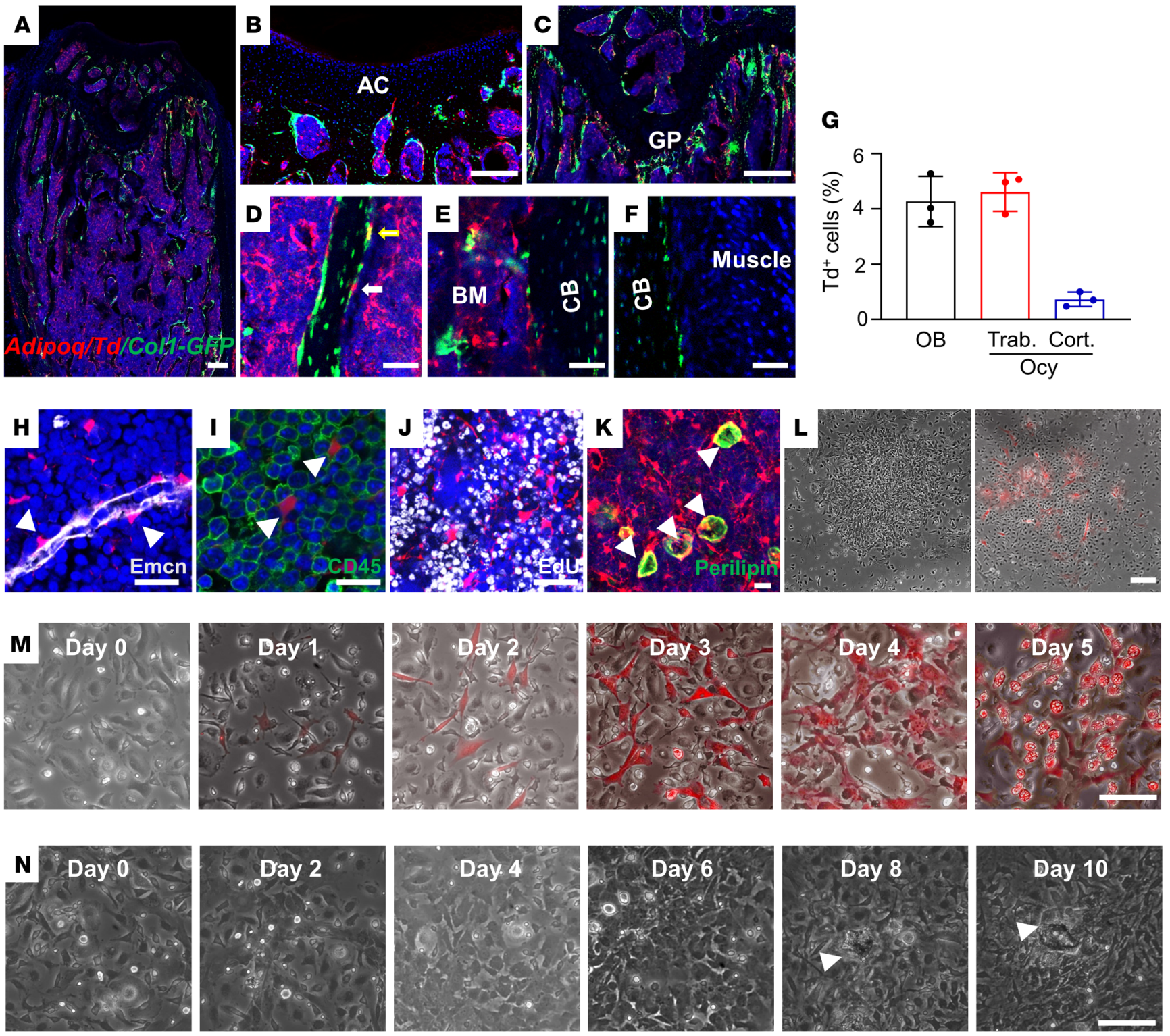

Figure 2. Adipoq-Cre labels MALPs in adult mouse bone marrow. (A) Representative low magnification fluorescence image of 3-month-old Adipoq/Td/ Col1-GFP mouse distal femur reveals many bone marrow Td ${ }^{+}$cells. Scale bar: $200 \mu \mathrm{m}$. (B-F) At a high magnification, Td does not label chondrocytes in articular cartilage (AC) (B) and growth plate (GP) (C), osteoblasts, nor osteocytes (D, E, F). White and yellow arrows point to $\mathrm{Td}^{+} \mathrm{GFP}^{-}$cells and Td+ $\mathrm{CFP}^{+}$cells at the bone surface, respectively. BM, bone marrow; CB, cortical bone. Scale bars: $200 \mu \mathrm{m}$ (B, C) and $50 \mu \mathrm{m}$ (D-F). (C) Quantification of Td+ cells in trabecular osteoblasts (OB) and osteocytes (Ocy) within trabecular (Trab.) and cortical (Cort.) bone ( $n=3$ mice/group). More than 1000 cells were counted per mouse. (H) Td labels pericytes (arrowheads) in bone marrow. Emcn, endomucin for vessel staining. (I) In Adipoq/Td mice, Td does not label CD45 ${ }^{+}$hematopoietic cells. (J) In vivo EdU injection reveals that bone marrow $\mathrm{Td}^{+}$cells do not proliferate. (K) All Perilipin ${ }^{+}$adipocytes (arrowheads) are $\mathrm{Td}^{+}$as well. Scale bar: $20 \mu \mathrm{m}$ (H-K). (L) CFU-F assay of bone marrow cells from Adipoq/Td mice shows that all CFU-F colonies are made of Td ${ }^{-}$cells (left image). Some Td ${ }^{+}$ cells did attach to the dish and form a small cluster within a Td- CFU-F colony (right image). Scale bar: $100 \mu \mathrm{m}$. (M) In vitro adipogenic differentiation of Td mesenchymal progenitors reveals that Td signal is turned on first followed by lipid accumulation. The same area was imaged daily by inverted fluorescence microcopy. Scale bar: $100 \mu \mathrm{m}$. (N) In vitro osteogenic differentiation of $\mathrm{Td}^{-}$mesenchymal progenitors reveals that Td signal remains off when a bony nodule forms. The same area was imaged daily by inverted fluorescence microcopy. Arrowheads point to a boney nodule. Scale bar: $100 \mu \mathrm{m}$.

trabecular number (Tb.N) and a 40\% decrease in trabecular separation (Tb.Sp) (Figure 4, H and I). Trabecular thickness (Tb.Th) and structure model index (SMI) remained the same. In contrast, structural parameters of cortical bone were not affected (Figure 4, $\mathrm{J}$ and $\mathrm{K}$ ). At 3 and 5 months of age, the high bone mass phenotype became more striking with 1.7- and 2.3-fold increases, respectively, in tibial trabecular BV/TV (Figure 4, H and I; and Supplemental
Figure 5, A and B). Cortical bone was still unaltered (Figure 4, J and K; and Supplemental Figure 5, C and D). Similar bone phenotypes were also observed in female mice (Supplemental Figure 6). Notably, trabecular BV/TV in 3-month-old female CKO mice was 2.9- and 2.0-fold higher than $W T$ at tibial and vertebral sites, respectively. For comparison, we constructed Dmp1-Cre Tnfsf11 fl/fl (RANKL-CKO ${ }^{D m p 1}$ ) mice to knockdown Tnfsf11 expression in osteo- 
A

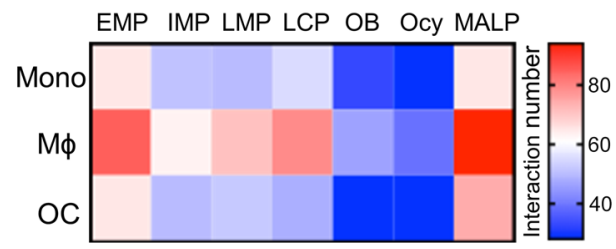

B

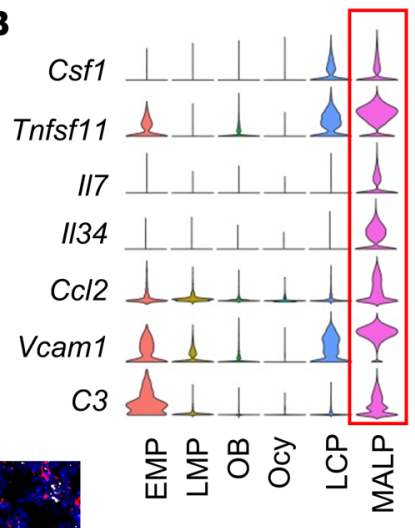

C

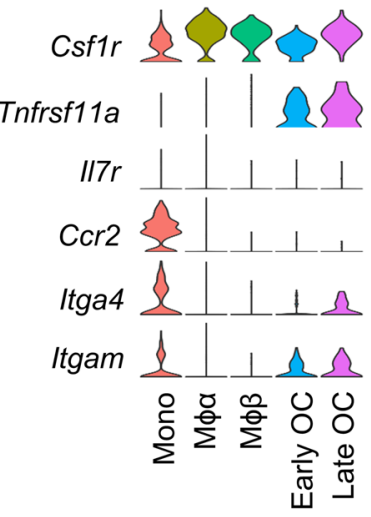

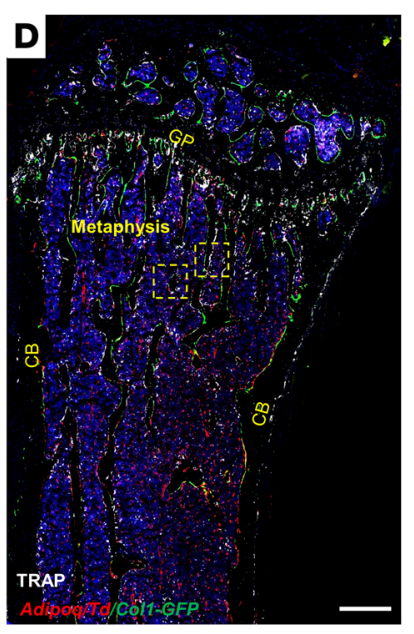

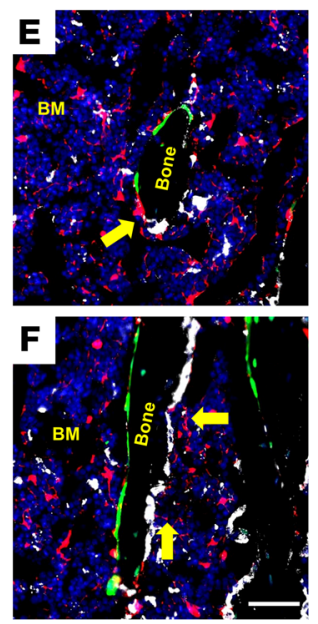

Figure 3. MALPs are the major producer of osteoclast regulatory factors in bone. (A) Ligand-receptor pair analysis of mesenchymal subpopulations with monocytes, macrophages, and osteoclasts. (B) Violin plots of osteoclast regulatory factors in mesenchymal subpopulations. (C) Violin plots of receptors for osteoclast regulatory factors in monocyte-macrophage lineage cells. (D) Representative fluorescence image of TRAP staining in 3-month-old Adipoq/Td/Col1-GFP mouse femur. Scale bar: $500 \mu \mathrm{m}$. (E, F) Enlarged images of boxed area in $\mathbf{D}$. Yellow arrows point to $\mathrm{Td}^{+}$cell processes touching nearby bone surface osteoclasts (white). Note that osteoblasts (green) and osteoclasts are often located at the opposite sides of bone (F). GP, growth plate; $\mathrm{CB}$, cortical bone; BM, bone marrow. Scale bar: $50 \mu \mathrm{m}$. cytes. In line with previous reports $(9,11)$, at 1 month of age, these mice displayed only an $18 \%$ increase in trabecular bone mass (Supplemental Figure 7). Taken together, our data suggest that MALPs contribute more to trabecular bone remodeling than osteocytes in young mice.

We next performed histology to analyze cellular changes in RANKL-CKO ${ }^{\text {Adipoq }}$ mice. Overall, WT mice had many more osteoclasts and osteoblasts at 1 month of age than at 3 months of age, indicating a higher bone turnover (Figure 5, A and B). TRAP staining revealed that osteoclast surface and number in the trabecular bone of 1-month-old RANKL-CKO Adipoq mice are decreased by $75 \%$ and 65\%, respectively, compared with WT mice. However, osteoclast formation at $\mathrm{COJ}$ and the endosteal surface of cortical bone was not changed, indicating that MALP-derived RANKL is not the decisive factor for osteoclastogenesis at those skeletal sites. To study whether RANKL deficiency affects osteoclast progenitors, we harvested bone marrow cells (BMCs) for in vitro osteoclastogenesis. BMCs from $W T$ and $C K O$ mice gave rise to the same quantity of multinucleated osteoclasts after M-CSF and RANKL induction (Supplemental Figure 8), indicating that MALP-derived RANKL does not affect the number of osteoclast progenitors.

Suppressed bone resorption in RANKL-CKOAdipoq mice leads to attenuated bone formation. Meanwhile, we observed that compared with WT mice, osteoblast number is significantly reduced by $52 \%$ and $41 \%$ in the trabecular bone of 1- and 3-month-old $R A N K L-C K O^{\text {Adipoq }}$ mice, respectively (Figure $5 \mathrm{C}$ ), whereas osteocyte density is not affected (Figure 5D). Double labeling showed a decrease in osteoblast activity (Figure 5E). Specifically, mineral apposition rate (MAR) and surface-referent bone formation rate $(\mathrm{BFR} / \mathrm{BS})$ were reduced by $55 \%$ and $58 \%$, respectively, in 1-month-old CKO mice (Figure $5 \mathrm{~F}$ ). Similar to osteoclasts, osteoblasts on endosteal cortical bone surface remained unchanged (Figure 5C). ELISA analysis of serum bone resorption (CTX-1) and formation (PINP) markers confirmed suppressed bone turnover in CKO mice (Figure 5G).

Osteoblasts are derived from bone marrow mesenchymal progenitors. To test whether those cells are affected in $C K O$ mice, we performed CFU-F assay. Strikingly, CFU-F frequency from bone marrow of 1-month-old CKO mice was drastically decreased by 67\% (Supplemental Figure 9A). However, once seeded, their growth curve was similar to those cells from WT mice (Supplemental Figure 9B). Furthermore, when subjected to differentiation, these progenitors exhibited similar levels of osteogenic and adipogenic differentiation, evidenced by lineage-specific staining (Supplemental Figure 9C) and marker gene expression (Supplemental Figure 9, D and E). These data implicate that suppressed bone resorption in $\mathrm{CKO}$ mice reduced the pool of bone marrow mesenchymal progenitors but did not affect their proliferative and differentiation abilities.

MALP-derived RANKL contributes to pathologic bone loss. To understand the functional role of MALP-derived RANKL in osteoclast-mediated bone resorption, we tested 2 mouse models of pathologic bone loss. In the calvaria of Adipoq/Td mice, $\mathrm{Td}^{+}$ cells were detected abundantly inside the bone marrow but not in the suture and periosteum (Figure 6A). All $\mathrm{Td}^{+}$cells had no lipid accumulation, indicating that they are MALPs. In the first model, we injected LPS above calvaria of 6-week-old mice to induce bone loss that mimics bacteria-induced bone loss. One week later, 
A

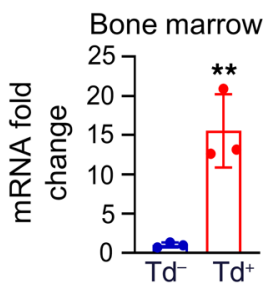

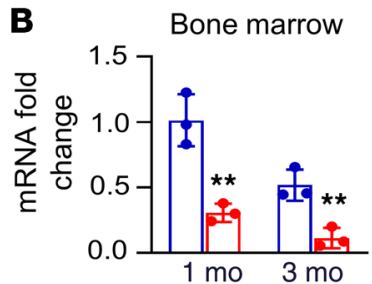
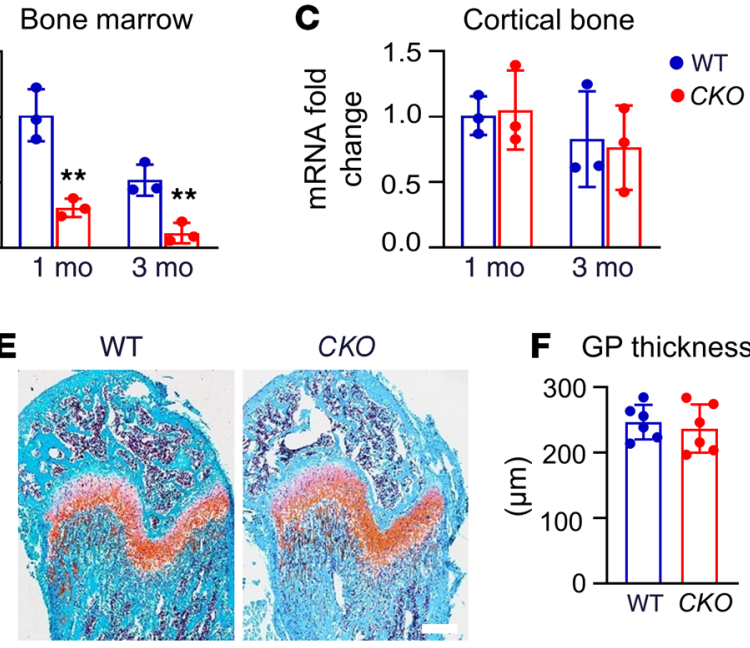

F GP thickness

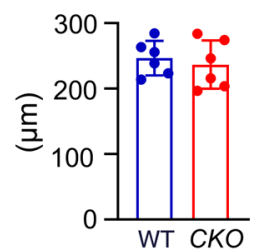

G Tibial length

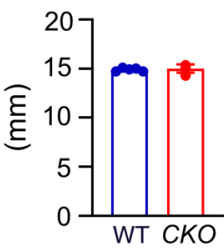

H

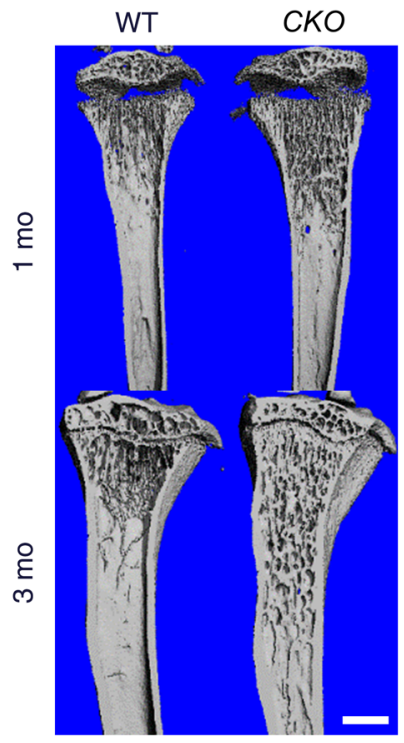

J

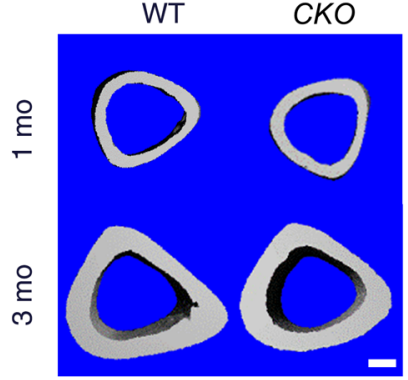

I
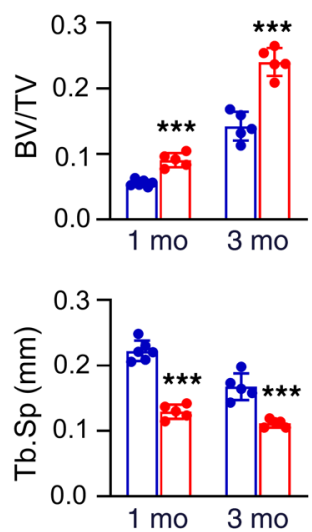

$\mathbf{K}$
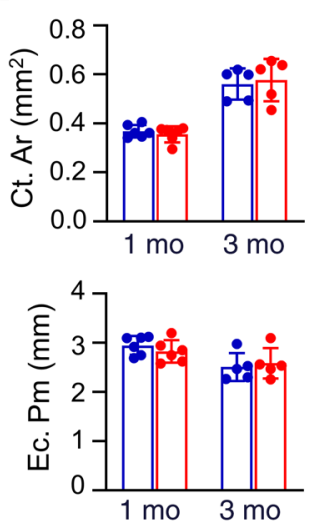
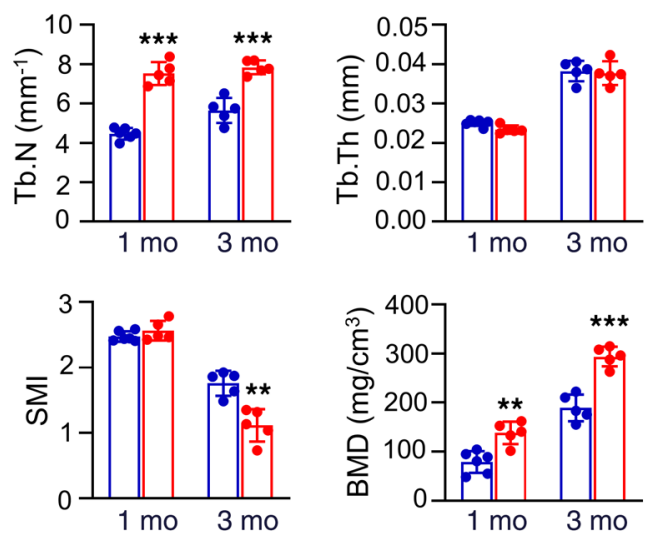

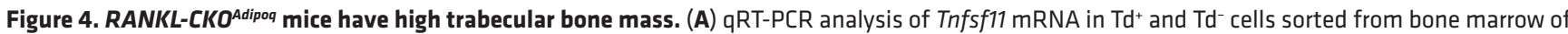
Adipoq/Td mice ( $n=3$ mice/group). (B) qRT-PCR analysis of Tnfsf11 mRNA in bone marrow of WT and RANKL-CKOAdipoq mice at 1 and 3 months of age ( $n=3$ mice/group). (C) qRT-PCR analysis of Tnfsf11 mRNA in cortical bone of WT and RANKL-CKOAdipou mice at 1 and 3 months of age ( $n=3$ mice/group). (D) Tooth eruption is not affected in RANKL-CKO Adipoq mice. (E) Representative Safranin 0/fast green staining of long bone sections from 1-month-old WT and RANKL-CKO Adipoq mice. Scale bar: $200 \mu \mathrm{m}$. (F) Quantification of femoral growth plate thickness ( $n=6$ mice/group). (C) Quantification of tibial length ( $n=6$ mice/group). (H) 3D microCT reconstruction of WT and RANKL-CKOAdipoq mouse tibiae reveals a drastic increase of trabecular bone at 1 and 3 months of age. Scale bar: $1 \mathrm{~mm}$. (I) MicroCT measurement of trabecular bone structural parameters from the secondary spongiosa region ( $n=5-6 \mathrm{mice} / \mathrm{group}$ ). (J) 3D microCT reconstructions of the tibial midshaft region. Scale bar: $0.2 \mathrm{~mm}$. (K) MicroCT measurement of cortical bone structural parameters from the midshaft region ( $n=5-6$ mice/group). ${ }^{* *} P<0.01$; ${ }^{* *} P<0.001 \mathrm{Td}^{+}$vs. $\mathrm{Td}^{-}$or $C K O$ vs. WT, 2-tailed unpaired Student's $t$ test. 
A
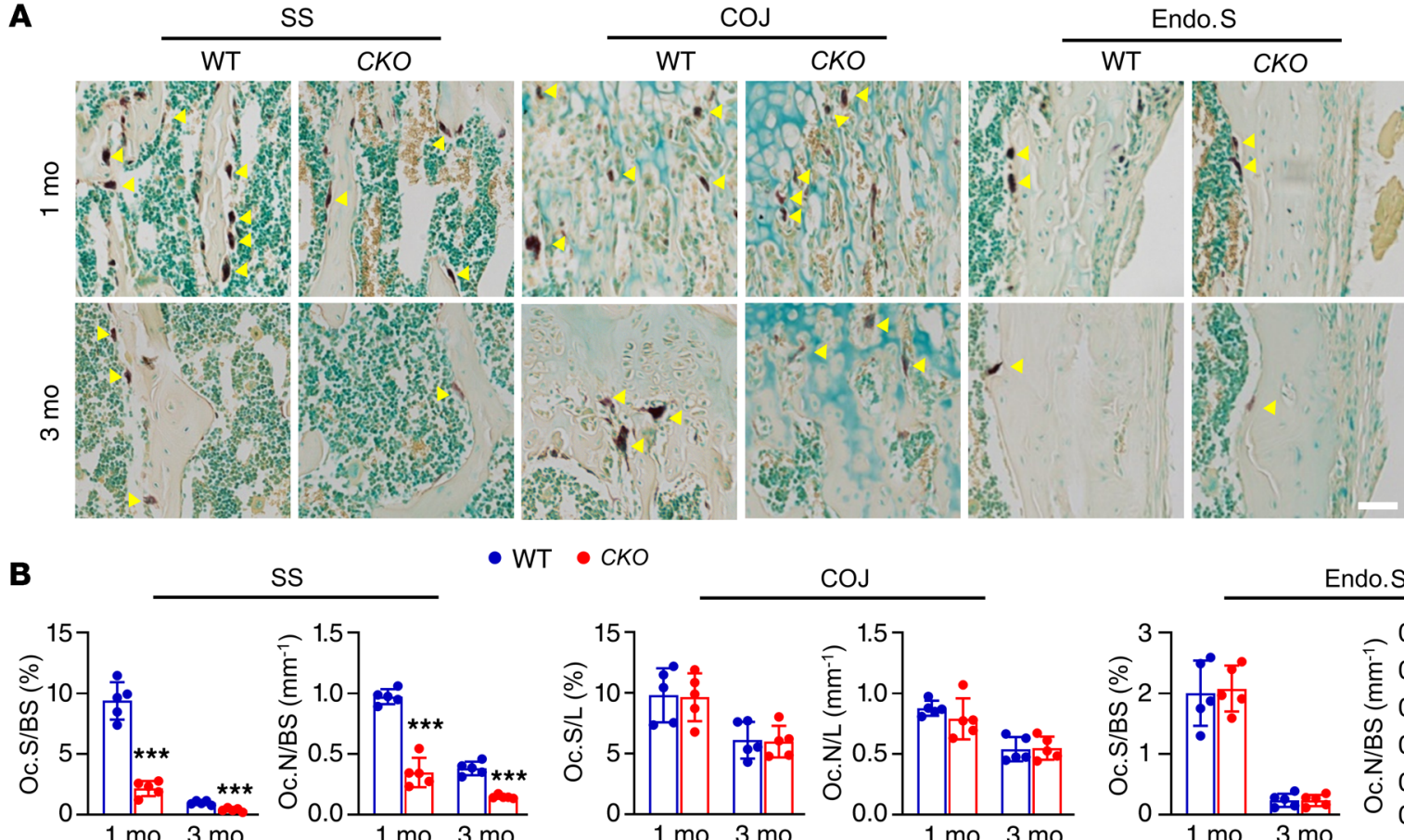

SS

- WT • CKO
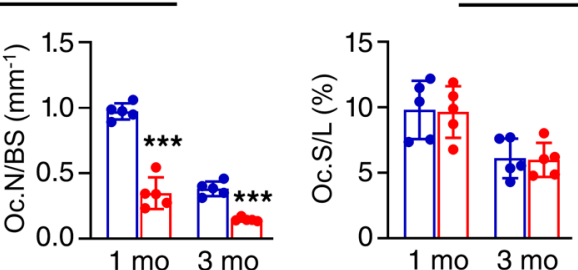

COJ

Endo.S

C
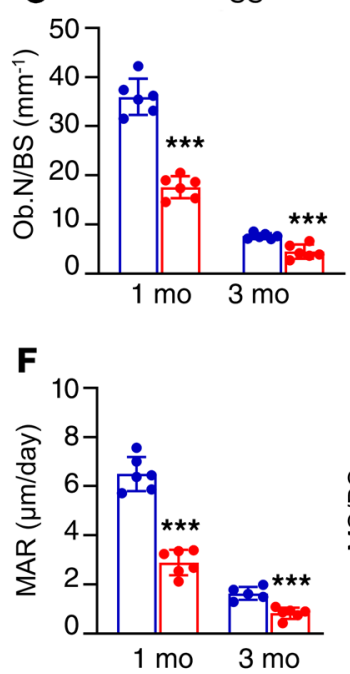

Endo.S

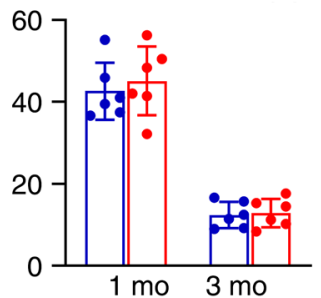

D

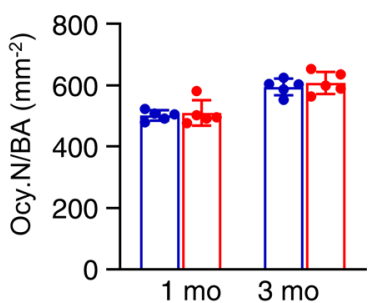

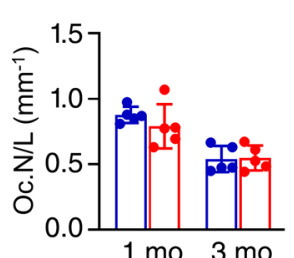
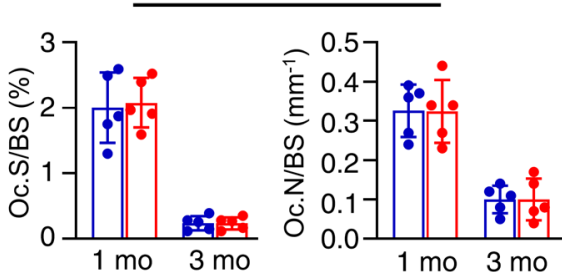

E

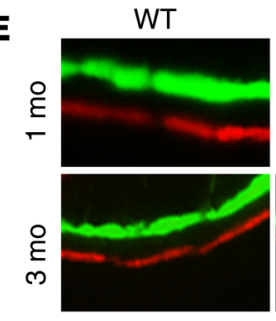

CKO

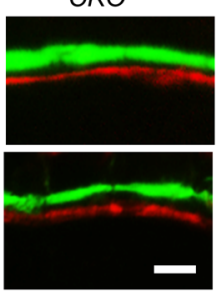

Figure 5. Bone resorption as well as bone formation are reduced in RANKL-CKO $^{\text {Adipoq }}$ mice. (A) Representative TRAP staining images show TRAP ${ }^{+}$osteoclasts (arrowheads) at different skeletal sites: secondary spongiosa (ss), COJ, and endosteal surface (Endo.S). Scale bar: $50 \mu \mathrm{m}$. (B) Quantification of osteoclast surface (Oc.S) and osteoclast number (Oc.N) at 3 skeletal sites ( $n=5-6$ mice/group). BS, bone surface; L, COJ length. (C) Quantification of osteoblast number (Ob.N) in the secondary spongiosa and at the endosteal surface ( $n=5-6$ mice/group). (D) Quantification of osteocyte density (osteocyte number per bone area, Ocy.N/BA) in the secondary spongiosa ( $n=5-6$ mice/group). (E) Representative double labeling in distal femurs of $W T$ and $C K O$ mice. Scale bar: $10 \mu \mathrm{m}$. (F) Bone formation activity is quantified ( $n=5-6 /$ group). (C) Serum ELISA analysis of bone resorption marker (CTX-1) and formation marker (PINP) in WT and CKO mice ( $n=5$ mice/group). ${ }^{*} P<0.05 ;{ }^{* *} P<0.01$; ${ }^{* *} P<0.001 C K O$ vs. WT, 2 -tailed unpaired Student's $t$ test.

we found a drastic increase of bone destruction in $W T$ calvaria but not in RANKL-CKO ${ }^{\text {Adipoq }}$ calvaria (Figure 6, B and C). TRAP staining revealed that LPS injections increased TRAP stained area and osteoclast number in $W T$ calvaria by 45 - and 34 -fold, respectively (Figure 6, D-G). In CKO mice, such increases were almost completely abolished.

In the second model, we performed ovariectomy (ovx) on female $C K O$ mice to mimic postmenopausal osteoporosis and examined vertebral trabecular bone 1.5 months later. Estrogen deficiency was confirmed by an $86 \%$ decrease in uterine weight in both WT and CKO mice (Supplemental Figure 10A). No body weight changes were observed (Supplemental Figure 10B). Ovx reduced trabecular BV/TV by $50 \%$ due to a $34 \%$ decrease in Tb.N and a 10\% decrease in Tb.Th in WT mice (Figure 7, A and B). CKO mice also showed a $30 \%$ reduction in BV/TV. Interestingly, while Tb.Th was similarly decreased, Tb.N remained the same. Histol- 
A

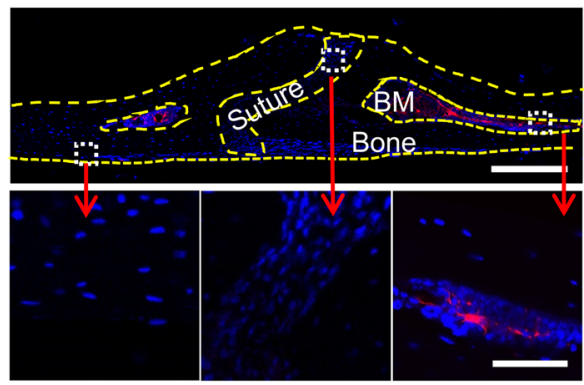

B

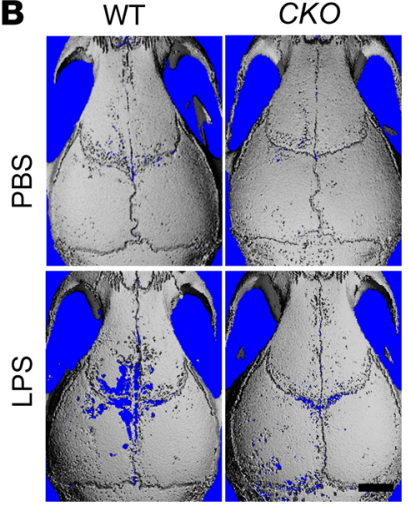

D

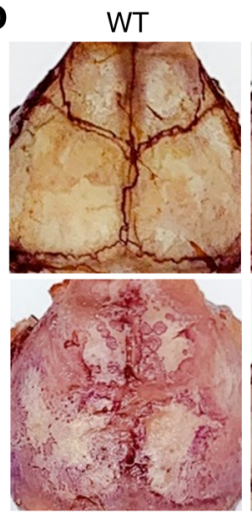

CKO
WT
$\mathbf{F}$

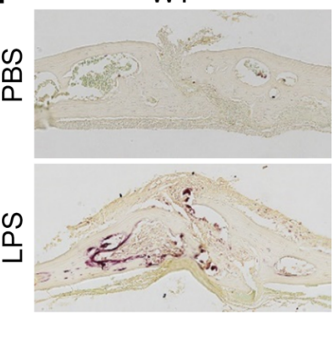

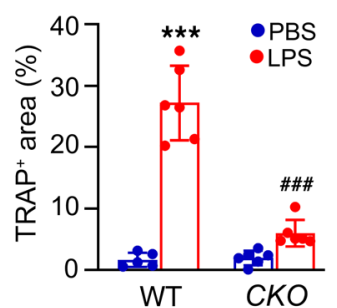
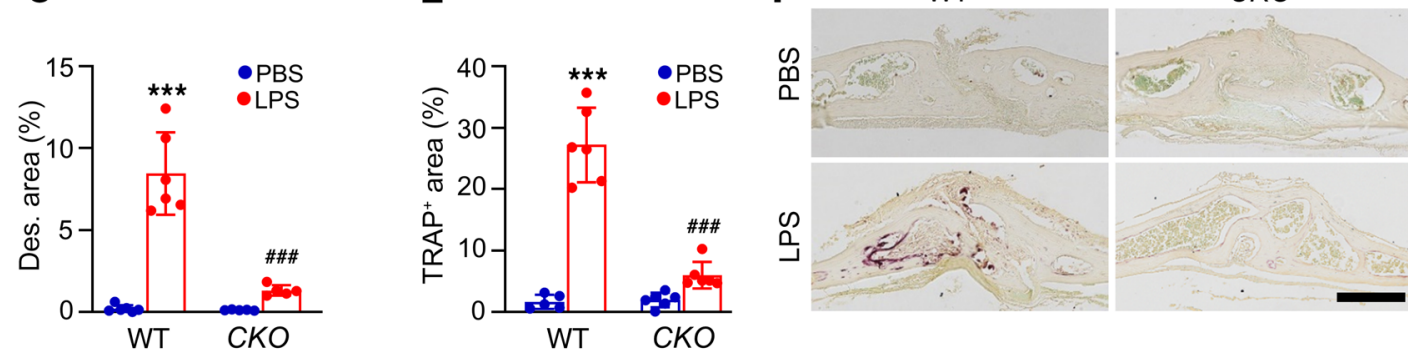

E

WT

CKO

Figure 6. RANKL-CKO ${ }^{\text {Adipoq }}$ mice are protected from LPS-induced calvarial bone lesions. (A) Representative coronal section of 1.5-month-old Adipoq/Td mouse calvaria. Bone surfaces are outlined by dashed lines. Boxed areas in the low magnification image (top) are enlarged to show periosteum (bottom left), suture (bottom middle), and bone marrow (BM, bottom right) regions. Scale bars: $200 \mu \mathrm{m}$ (top) and $20 \mu \mathrm{m}$ (bottom). (B) Representative 3D microCT reconstruction of mouse calvaria at 1 week after vehicle (PBS) or LPS injection. Scale bar: $2 \mathrm{~mm}$. (C) Quantification of percentages of bone destruction area (Des. area) in calvaria ( $n=6$ mice/group). (D) Representative images of TRAP staining of whole calvaria. Scale bar: 2 mm. (E) Quantification of percentages of TRAP+ area in calvaria ( $n=6$ mice/group). (F) Representative images of calvaria coronal section stained by TRAP. Scale bar: 2 mm. (G) Quantification of osteoclast number (Oc.N) in calvaria ( $n=6$ mice/group). ${ }^{* *} P<0.001$, LPS vs. PBS; \#\#\# $P<0.001$, CKO vs. WT, 2 -way ANOVA with Tukey's post hoc analysis.

ogy revealed that increases of osteoclast number and surface are much greater in $W T$ mice ( $118 \%$ and $82 \%$, respectively) than in CKO mice ( $81 \%$ and $45 \%$, respectively, Figure 7, C and D). Meanwhile, osteoblast activity, as measured by MAR and BFR/BS, was increased in both genotypes after ovx (Figure 7, E and F). Taken together, the above data demonstrate that RANKL from MALPs is primarily responsible for osteolytic lesions in the LPS treatment model and partially responsible for enhanced bone resorption in the ovx model.

\section{Discussion}

Bone remodeling, a balance between osteoblastic bone formation and osteoclastic bone resorption, is critical for the maintenance of skeletal structural integrity and mineral homeostasis. It has long been conceived that osteogenic cells are the major support cells for osteoclastogenesis, and thus they promote bone resorption. In this work, we demonstrated that the committed adipogenic precursors, MALPs, are another important player in controlling bone resorption. In silico analysis revealed that among mesenchymal subpopulations, they have the most interactions with monocyte-macrophage lineage cells, and predicted that they express critical osteoclast regulator factors, including RANKL, at a much higher level than other mesenchymal cells (e.g., osteoblasts and osteocytes) in young and adult mice. Strikingly, adipogenic-specific knockdown of RANKL caused a dras- tic high bone mass phenotype (60\%-70\% increase in BV/TV) as early as 1 month of age. In contrast, at the same age, mice with osteocyte-specific deficiency of RANKL showed only small bone changes in our hands (an 18\% increase in BV/TV) or no changes in other groups $(9,11)$. Furthermore, MALP-derived RANKL is absolutely required for LPS-induced osteolysis and partially required for ovx-induced bone loss, reinforcing the importance of MALPs as an important cellular regulator of bone remodeling under normal and diseased conditions.

Past studies have provided mostly in vitro evidence that bone marrow adipocytes support osteoclast formation. Using an elegant inverted coculture method, Goto et al. showed that primary human bone marrow LiLAs stimulate $\mathrm{TRAP}^{+}$multinucleated osteoclast formation in the presence of TNF- $\alpha$ or dexamethasone via upregulation of RANKL $(37,38)$. Adipogenic differentiation of mouse bone marrow mesenchymal progenitors was found to be associated with increased expression of RANKL and decreased expression of OPG, a decoy receptor of RANKL (39). Further studies showed that adipogenic TFs C/EBP $\beta$ and $\delta$ activate RANKL gene transcription. Interestingly, mesenchymal progenitors from aged mice are better at supporting osteoclast formation in coculture than those from young mice when using adipogenic differentiation medium. This is consistent with the well-known effects of aging on bone marrow adiposity and our finding that aging expands the MALP population (12). A recent study careful- 
A

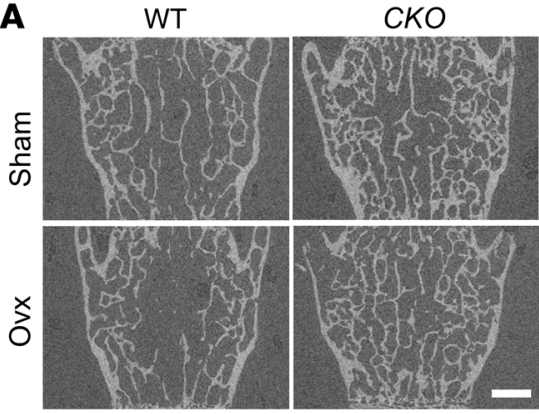

C

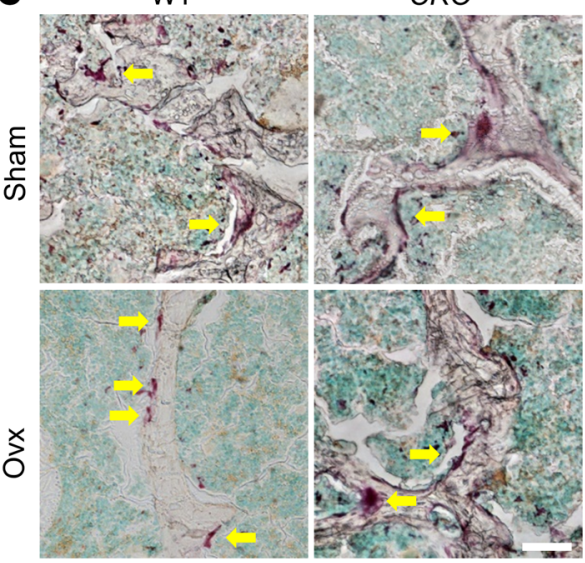

E

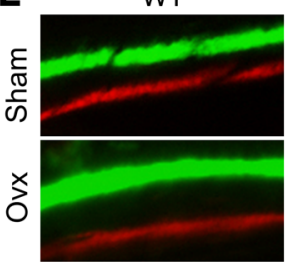

CKO

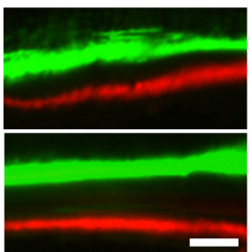

B

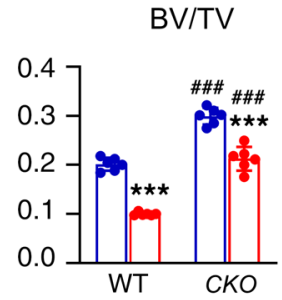

Tb.Sp (mm)

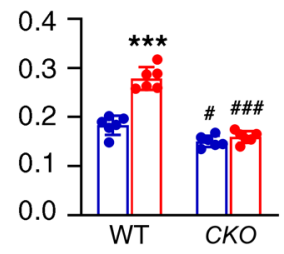

D

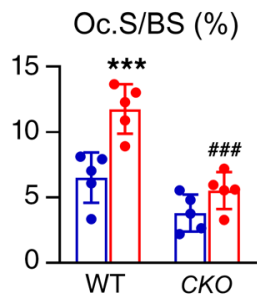

$\mathbf{F}$

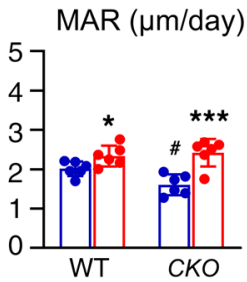

Tb. N $\left(\mathrm{mm}^{-1}\right)$

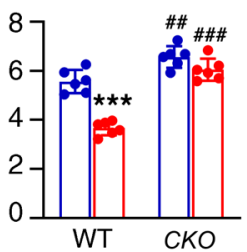

SMI

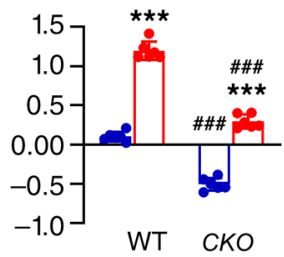

Oc.N/BS $\left(\mathrm{mm}^{-1}\right)$

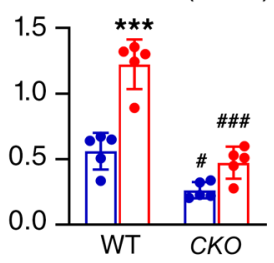

-Sham

- Ovx

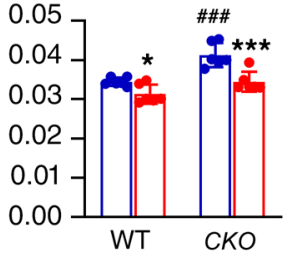

$\mathrm{BMD}\left(\mathrm{mg} / \mathrm{cm}^{2}\right)$

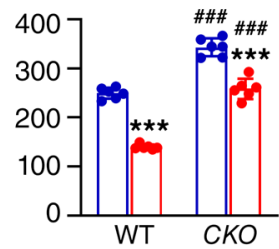

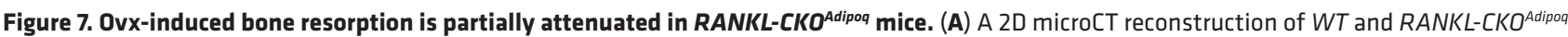
mouse vertebrates at 1.5 months after sham or ovx surgery. Scale bar: $500 \mu \mathrm{m}$. (B) MicroCT measurement of trabecular bone structural parameters in vertebrates ( $n=5-6$ mice/group). (C) Representative image of TRAP staining in vertebrate after sham or ovx surgery. Arrows point to TRAP+ osteoclasts. Scale bar: $100 \mu \mathrm{m}$. (D) Quantification of osteoclast surface (Oc.S/BS) and number (Oc.N) in vertebrates after surgery ( $n=5-6$ mice/group). (E) Representative double labeling in WT and $C K O$ vertebrates after surgery. Scale bar: $10 \mu \mathrm{m}$. (F) Bone formation activity is quantified ( $n=5-6$ mice/group). ${ }^{*}<0.05$, ${ }^{* *} P<0.001$, Ovx vs. Sham; ${ }^{\#} P<0.05,{ }^{*} P<0.01,{ }^{\# \#} P<0.001, C K O$ vs. WT, 2 -way ANOVA with Tukey's post hoc analysis.

ly dissected adipocytes derived from bone marrow mesenchymal progenitors in culture into nonlipid-laden and lipid-laden ones, which bear resemblance to MALPs and LiLAs, respectively, in our analysis. A striking finding was that RANKL is mostly presented in non-lipid-laden adipocytes but not in lipid-laden adipocytes (40). Since scRNA-seq cannot capture LiLAs, we do not know whether Tnfsf11 expression is downregulated when MALPs become LiLAs. However, these in vitro data as well as the high ratio of MALPs vs. LiLAs in vivo strongly indicate that MALPs are the major cell type controlling bone resorption in vivo. Further, here we provided what we believe is the first in vivo evidence that MALP-derived RANKL controls trabecular bone remodeling.

Marrow adipose tissue (MAT) is a unique adipose tissue that is morphologically and functionally distinct from peripheral adipose tissues (41). Traditionally, it only refers to LiLAs. Our discovery of MALPs adds another important and abundant cell population to this tissue although no counterpart of MALPs has been identified in other adipose tissues. Hence, it is not surprising that MAT possesses a set of functions that does not exist in other adipose tissues. Our previous study revealed that MALPs maintain bone marrow vessel integrity and inhibit bone formation (12). Here we show that MALPs promote bone resorption. In line with our results, it was reported that only bone marrow adipocytes but not peripheral adipocytes express RANKL (42). The unique functionality attributable to MAT is likely due to its special location in bone marrow, where bone remodeling and hematopoiesis occur constantly. Having a cell body and multiple processes, MALPs form a 3D network structure that contacts almost every cell inside the bone. Hence, we propose that their main function is to regulate the bone marrow environment, including bone resorption.

It is well-known that bone constantly undergoes remodeling to preserve skeletal integrity and to regulate mineral homeostasis. 
Bone remodeling initiates with bone resorption via osteoclasts followed by bone formation via osteoblasts at the same site. Our data provided 2 perspectives toward this process. First, since MALPs but not osteoblasts extend cell-cell contact with osteoclasts in bone, the initiation signal of bone remodeling might partially come from MALPs that abundantly express Tnfsf11. Interestingly, our sequencing data set revealed that Tnfsf11 expression in MALPs was modestly decreased in 3-month-old mice compared with that in 1-month-old mice (Supplemental Figure 11), which correlated well with the reduced bone turnover in adult mice compared with young mice. Second, our results clearly demonstrated that osteoclasts played a critical role in promoting osteoblast formation. RANKL deficiency in MALPs not only drastically reduced osteoclast formation but also reduced osteoblast number and suppressed bone formation activity. These data are consistent with previous discoveries that osteoclasts talk back to osteoblasts, such as the reverse signaling of RANKL/RANK (43) and the forward signaling of Ephrin2/EphB4 (44), indicating that osteoblast formation during the second step of bone modeling is likely promoted by active osteoclasts.

Our studies relied on the specificity of Adipoq-Cre for adipogenic cells only but not for mesenchymal progenitors or osteogenic cells. A prior report stated that Adipoq-Cre targets $1.4 \%$ and $21 \%$ of osteoblasts at 4 and 24 weeks of age, respectively, based on flow analysis of $\mathrm{Alpl}^{+}$cells (45). Immunofluorescence images also showed some $\mathrm{Td}^{+}$cells at the bone surface. According to our sequencing data, Alpl is expressed in MALPs albeit at a lower level than osteoblasts (data not shown). Therefore, this study might overestimate the overlap between Adipoq ${ }^{+}$cells and osteoblasts. In Adipoq/Td/Col-GFP mouse bones, we also observed some $\mathrm{Td}^{+}$ cells on the bone surface but the majority of them were $\mathrm{GFP}^{-}$, indicating that they were not mature osteoblasts. Moreover, since the vast majority of osteocytes, which are much easier to define morphologically than osteoblasts, were not $\mathrm{Td}^{+}$, we concluded that Adipoq-Cre is specific to adipogenic cells at least up to 3 months of age. We previously reported that Dmp1 is also expressed in LCP, the mesenchymal progenitor subpopulation right before adipogenic and osteogenic differentiation (12). To validate that, we found that $\mathrm{Td}^{+}$cells from Dmp1-Cre $\mathrm{Td}$ mice label almost all osteoblasts and osteocytes, some stromal cells, and a few Perili$\mathrm{pin}^{+}$adipocytes, indicating that Dmp1-Cre starts to label a portion of MALPs at 3 month of age (12). In the future, inducible systems, such as Adipoq-CreER and Dmp1-CreER, are required to compare the relative contribution of adipogenic and osteogenic cells to bone resorption in adult and old mice.

While our single-cell data set and subsequent animal studies identified MALPs as the major support cells for osteoclastogenesis, we cannot rule out the importance of other cells. One limitation of our single-cell approach is that our data set might contain only young osteocytes but not mature ones because $\mathrm{Td}^{+}$cells were collected via enzymatic digestion of bones longitudinally cut into half after flushing out bone marrow. Since Tnfsf 11 mRNA is more than 10 times higher in osteocytes than in osteoblasts (10), it is likely that mature osteocytes play a more important role in controlling osteoclastogenesis than young, surface osteocytes. Interestingly, RANKL-CKO $O^{\text {Adipoq }}$ mice displayed reduced bone resorption only within trabecular bone but not at cortical bone. Since
MALPs do not exist in the periosteum, it is expected that periosteal bone resorption is not affected in CKO mice. However, MALPs are abundant at the endosteal surface. To reconcile these data, we propose that mature osteocytes, which are abundant in cortical bone but relatively scarce in trabecular bone, control osteoclast formation at the surface of cortical bone. In line with this idea, Xiong et al. found that RANKL-CKO ${ }^{D m p 1}$ mice are resistant to tail suspension-induced cortical bone loss (9). Similarly, MALPderived RANKL does not contribute to cartilage-to-bone remodeling during endochondral ossification even though MALPs are abundant at COJ. Hypertrophic chondrocytes control osteoclast formation in this event (9). Moreover, during estrogen deficiency, RANKL produced from B cells and osteocytes, in addition to MALPs, is required for the enhanced osteoclastic activity $(46,47)$. Our studies also demonstrated a dominant action of MALPs in LPS-induced osteolysis in calvaria. Collectively, osteoclast formation is controlled by a variety of cells in bone in skeletal site-specific and disease-dependent manners (graphical abstract).

Our scRNA-seq data set and analysis have another limitation. Since our original purpose was to isolate mesenchymal lineage cells, the Col2/Td mouse model was adopted. However, for reasons we do not yet understand, a small number of hematopoietic cells were also $\mathrm{Td}^{+}$. One theory we have is that due to the extremely low expression of Col2a1 in hematopoietic cells, only a few of them express Cre at a level high enough to trigger the deletion of the STOP codon in the Td promoter. Meanwhile, we used this fact to our advantage for the subsequent monocyte-macrophage lineage and cell-cell interaction analyses. Since we did not specifically select myeloid cells for analysis, it is possible that hematopoietic cells in our data set did not contain all subsets of monocytes, macrophages, and osteoclasts. Therefore, the pseudotime trajectory analysis might not be inclusive. Moreover, recent studies have demonstrated that tissue-resident macrophages contain both embryonic stage-derived macrophages and adult HSCderived macrophages (48) and that osteoclasts in adult bone are also a fusion of cells originated from these 2 types of macrophages $(49,50)$, raising a concern about whether scRNA-seq analysis could distinguish these 2 sources of progenitors. Nevertheless, our analysis revealed that $\mathrm{M} \phi \alpha$ cells differentiate into 2 types of terminal cells: osteoclasts that eat bone matrix and $\mathrm{M} \phi \beta$ that eat apoptotic cells in bone. $\mathrm{M} \phi \alpha$ cells could be a combination of embryonic stage-derived and HSC-derived macrophages. Interestingly, they express a variety of cytokines and chemokines, in accordance with previous findings that bone marrow macrophages regulate hematopoiesis and bone formation (51).

It would be interesting to explore the action of MALPs in other bone disorders and therapies. Parathyroid hormone (PTH1-34) is an FDA-approved drug for improving bone mass in patients with osteoporosis (52). Ablation of its receptor in skeletal mesenchymal progenitors using Prx1-Cre leads to increased RANKL expression in bone marrow adipocytes, enhanced marrow adiposity, and bone resorption (42), implying a role of MALPs in the anabolic actions of PTH.

The current mainstay drugs for osteoporosis are antiresorptive agents, such as bisphosphonates and anti-RANKL antibody (denosumab) (53). However, their long-term use has led to concern for undesired effects, such as atypical fracture and osteone- 
crosis. MALPs not only produce RANKL but also other adipokines and cytokines that have osteoclast regulatory actions. Furthermore, ablation of this cell population causes the most profound bone formation we have ever observed, indicating a pivotal role in regulating bone generation and regeneration (12). In the future, understanding the mechanisms of the dual actions of MALPs on bone remodeling and seeking novel approaches to target this cell population could be of critical value in developing new therapies for osteoporosis and other disorders of pathologic bone loss.

\section{Methods}

Animal study design. All mouse strains used in this study were maintained on C57BL/6 background. Col2-Cre Rosa-tdTomato (Col2/ Td), Adipoq-Cre Rosa-tdTomato (Adipoq/Td) mice were generated by breeding Rosa-tdTomato (Jackson Laboratory) mice with Col2-Cre (54) and Adipoq-Cre (55) mice, respectively. Adipoq-Cre Rosa-tdTomato 2.3kbCol1-GFP (Adipoq/Td/Col1-GFP) were generated by breeding Adipoq/Td mice with 2.3kbCol1-GFP mice (29).

Mice carrying floxed Tnfsf 11 exons 3 and 4 were constructed by homologous recombination at the Transgenic and Chimeric Mouse Facility (University of Pennsylvania, Philadelphia, Pennsylvania, USA). The cloning strategy is depicted in Supplemental Figure 12. A Tnfsfl1 gene sequence containing exons 3 and 4 flanked by FRT and loxP sites was inserted in the pLFNeoLoxP targeting vector containing the $3^{\prime}$ recombination arm. The resultant targeting vector was confirmed by sequencing and used for generating Tnfsfl1floxed ES cells and mice $(C 57 B L / 6)$. To eliminate the interference of the neomycin cassette, mice carrying the targeted allele were crossed with C57BL/6 background FLPe mice (Jackson Laboratory) to generate $T n f s f 11^{l l}$ mice. Next, these mice were mated with $\mathrm{CMV}$-Cre mice to confirm their genotype. For genotyping by PCR, the following primers were used: P1, 5'-ACTTTTTAAGCATTTTGGGAGCTC-3'; P2， 5'-AGTATTTACTTTATTAGACCAGAGACC-3'; P3, 5' - TGGTTTCCAAACTAATCTACTTCG-3'; CMV-Cre F, 5' -GCGGTCTGGCAGTAA AAACTATC-3'; CMV-Cre R, 5'-GTGAAACAGCATTGCTGTCACTT-3'; CMV-Cre internal positive control forward, 5'-CTAGGCCACAGAATT GAAAGATCT-3'; CMV-Cre internal positive control reverse, 5'-GTAGGTGGAAATTCTAGCATCATCC-3'.

To generate RANKL-CKO ${ }^{A d i p o q}$ mice, we first bred Adipoq-Cre with Tnfsf $11^{f / f l}$ mice to obtain Adipoq-Cre Tnfsf $11^{f / /+}$, which were then crossed with $T n f s f 11^{f / f l}$ to generate RANKL-CKO $O^{\text {Adipoq }}$ mice and WT (Tnfsf $11^{f / f l}$ and $\left.T n f s f 11^{f /+}\right)$ siblings. RANKL-CKO ${ }^{D m p 1}$ mice were generated using a similar breeding strategy with Dmp1-Cre (56).

For LPS-induced bone destruction, 6-week-old male mice were injected with $25 \mathrm{mg} / \mathrm{kg}$ LPS (MilliporeSigma) or PBS above calvaria. After 7 days, calvariae were collected and analyzed by microCT followed by TRAP staining.

For ovx-induced bone destruction, ovx or sham operation was performed on 3-month-old female mice. Six weeks later, L4 and L5 vertebrae were collected and analyzed by microCT followed by dynamic histomorphometry and TRAP staining.

Hematopoietic phenotyping of bone marrow cells. Bone marrow was flushed from femurs of 1-month-old Col2/Td mice. The lineage cell compartment of the bone marrow was analyzed by staining for myeloid (rat anti-mouse Gr-1 APC-Cy7, BD Biosciences, catalog 557661, rat anti-mouse Mac-1 APC, eBioscience, catalog 17-0112-83), lymphoid (rat anti-mouse B220 FITC, eBioscience, catalog 11-0452-82; hamster anti-mouse CD3 PE-Cy7, eBioscience, catalog 25-0031-82), erythroid (rat anti-mouse Ter119 APC, BD Biosciences, catalog 557909), or megakaryocytes (rat anti-mouse CD41 FITC, BD Biosciences, catalog 553848). The HSPC compartment was analyzed by staining for lineage (biotin-Ter-119, -Mac-1, -Gr-1, -CD4, -CD8 $\alpha$, -CD5, -CD19, and -B220, eBioscience, catalog numbers 13-5921-85, 13-0051-85, 13-5931-86, 13-0112-86, 13-0452-86, 13-0041-86, 13-0081-86, and 13-0193-86, respectively) followed by staining with streptavidin-PE-TexasRed (Invitrogen, catalog SA1017), rat anti-mouse cKit APC-Cy7 (eBioscience, catalog 47-1171-82), rat anti-mouse Sca1 PerCP-Cy5.5 (eBioscience, catalog 45-5981-82), hamster anti-mouse CD48 APC (eBioscience, catalog 17-0481-82), and rat anti-mouse CD150 PE-Cy7 (BioLegend, catalog 115914). All flow cytometry experiments were performed on a BD Biosciences LSR Fortessa flow cytometer and analyzed by FlowJo v10.5.3 for MAC.

Single-cell RNA sequencing of endosteal bone marrow cells. We constructed 3 batches of single-cell libraries of endosteal $\mathrm{Td}^{+}$bone marrow cells from 1-month-old $(n=2), 1.5$-month-old $(n=3)$, and 3-month-old $(n=3)$ male Col2/Td mice for sequencing as previously described (12). Sorted $\mathrm{Td}^{+}$cells were loaded in Chromium controller (V2 chemistry version, 10X Genomics), barcoded, and purified as described by the manufacturer, and sequenced using a $2 \times 150$ pair-end configuration on an Illumina HiSeq platform at a sequencing depth of approximately 400 million reads. Cell ranger (version 3.0.2, https://support. 10xgenomics.com/single-cell-geneexpression/software/pipelines/ latest/what-is-cell-ranger) was used to demultiplex reads, followed by extraction of cell barcode and UMIs. The cDNA insert was aligned to a modified reference mouse genome ( $\mathrm{mm} 10)$.

Seurat package v3 was used for filtering, variable gene selection, dimensionality reduction analysis, and standard clustering (57). Doublets or cells with poor quality (genes $>6000$, genes $<200$, or $>5 \%$ genes mapping to mitochondrial genome) were excluded. Expression was natural log transformed and normalized for scaling the sequencing depth to a total of $1 \times 10^{4}$ molecules per cell. For the integrated data set, anchors from different data sets were defined using the FindIntegrationAnchors function, and these anchors were then used to integrate data sets together with IntegrateData. Data sets were scaled by regressing out the number of UMIs and percentage of mitochondrial genes. Statistically significant principle components were selected as input for UMAP plots. Different resolutions for clustering were used to demonstrate the robustness of clusters. Cell-cycle analysis were calculated by using the Seurat cellcycle scoring function, proliferative cells were defined as cells in G2M or S phase. Data sets were further scaled by regressing out G2M.Score and S.Score. In addition, differentially expressed genes (DEGs) within each cluster relative to the remaining clusters were identified using FindMarkers within Seurat. Subclustering was performed by isolating the monocytic lineage clusters using known marker genes, followed by reanalysis as described above. GO terms and clusters, as well as KEGG pathway enrichment, were identified using the database for annotation, visualization, and integrated discovery (DAVID) (58).

To computationally delineate the developmental progression of monocytes, macrophages, and osteoclasts and order them in pseudotime, we used the algorithms implemented in the Monocle 2 package. We ordered cells by selecting genes with high dispersion across cells, using a parameter of "mean_expression $\geq 0.05$ and dispersion_empirical $\geq 1$ * dispersion_fit." Lists of genes were selected for dimensional reduction to generate the trajectory reconstruction using the nonlin- 
ear reconstruction algorithm DDRTree. Branched expression analysis modeling (BEAM) was used to determine the genes that are differentially expressed between branches. A mouse TF list (TFdb) was used to detect 64 TFs among the DEGs (59).

To analyze cell-cell communication mediators among different cell types, we used CellPhoneDB, a repository of ligands, receptors, and interaction data that relies on public information to annotate receptors and ligands (60). Briefly, each cell type cluster defined by Seurat was used as input into CellPhoneDB to generate interaction numbers of the ligand/receptor pair among different groups based on statistical methods and analyses.

Micro-computed tomography (microCT) analysis. MicroCT analysis (microCT 35, Scanco Medical AG) was performed at $6 \mu \mathrm{m}$ isotropic voxel size as previously described (14). Briefly, the proximal end of the tibia corresponding to a $0 \mathrm{~mm}$ to $2.8 \mathrm{~mm}$ region below the growth plate was scanned at $6 \mu \mathrm{m}$ isotropic voxel size to acquire a total of 462 $\mu \mathrm{CT}$ slices per scan. The images of the secondary spongiosa regions (0.6-1.8 mm below the lowest point of the growth plate) were contoured for trabecular bone analysis. At the tibia midshaft, a total of 100 slices located $4.8 \mathrm{~mm}$ to $5.4 \mathrm{~mm}$ away from the proximal growth plate were acquired for cortical bone analyses by visually drawing the volume of interest (VOI). In vertebrae, the region (total about 300 slices) 50 slices away from the top and bottom end plates was acquired for trabecular bone analysis. The trabecular bone tissue within the VOI was segmented from soft tissue using a threshold of $487.0 \mathrm{mgHA} / \mathrm{cm}^{3}$ and a Gaussian noise filter ( bone tissue was segmented using a threshold of $661.6 \mathrm{mgHA} / \mathrm{cm}^{3}$ and a Gaussian noise filter ( igma $=1.2$, support $=2.0$ ). A 3D standard microstructural analysis was performed to determine the geometric trabecular BV/TV, bone mineral density (BMD), Tb.Th, Tb.Sp, Tb.N, and SMI. For analysis of cortical bone, periosteal perimeter (Ps.Pm), endosteal perimeter (Ec.Pm), cortical bone area (Ct.Ar), cortical thickness (Ct.Th), polar moment of inertia (pMOI), and tissue mineral density (TMD) were recorded. All calculations were performed based on 3D standard microstructural analysis (61).

Calvaria microCT analysis was performed at $15 \mu \mathrm{m}$ isotropic voxel size. The 3D images were reconstructed to visualize the destructive area. A square region of $8 \mathrm{~mm} \times 8 \mathrm{~mm}$ centered at the midline suture was selected for further quantitative analysis by ImageJ.

Histology and bone histomorphometry. To obtain whole-mount sections for immunofluorescence imaging of Adipoq/Td/Col1-GFP mouse bones, freshly dissected femurs were fixed in 4\% PFA for 1 day, decalcified in 10\% EDTA for 4-5 days, and then immersed into $20 \%$ sucrose and $2 \%$ polyvinylpyrrolidone (PVP) at $4{ }^{\circ} \mathrm{C}$ overnight. The samples were embedded into medium containing $8 \%$ gelatin, $20 \%$ sucrose, and $2 \%$ PVP and sectioned at $50 \mu \mathrm{m}$ in thickness. Sections were incubated with rat anti-CD45 (BioLegend, catalog 103101), rat anti-Endomucin (Santa Cruz, catalog sc-65495), or rabbit anti-Perilipin (Cell Signaling, catalog 9349) at $4^{\circ} \mathrm{C}$ overnight followed by incubation with Alexa Fluor 647 antirat (Abcam, ab150155) or anti-rabbit (Abcam, ab150157) secondary antibodies for 1 hour at room temperature. For EdU staining, mice received $1.6 \mathrm{mg} / \mathrm{kg}$ EdU 1 day and 3 hours before sacrifice and the staining was carried out according to the manufacturer's instructions (Thermo Fisher Scientific, Click-iT EdU Alexa Fluor 647 Imaging Kit, D3822).

To obtain paraffin sections, mouse bones were fixed in $4 \%$ PFA for 24 hours and decalcified in a 10\% EDTA for 3-4 weeks for long bones and vertebrates and 5-7 days for calvariae at $4^{\circ} \mathrm{C}$. Samples were then embedded in paraffin, sectioned at $6 \mu \mathrm{m}$ in thickness, and processed for H\&E staining, Safranin O/fast green staining, or tartrate-resistant acid phosphatase (TRAP) staining using a kit (MilliporeSigma, catalog 387A).

To obtain cryosections, mouse bones were dissected and fixed in $4 \%$ PFA for 24 hours, dehydrated in 30\% sucrose in PBS, embedded in OCT compound, and sectioned at $6 \mu \mathrm{m}$ in thickness using a cryofilm tape (Section Lab). Fluorescence TRAP staining was performed as previously described (62). Briefly, sections were incubated in TRAP buffer $(0.92 \%$ sodium acetate anhydrous, $1.14 \% \mathrm{~L}-(+)$ tartaric acid, $1 \%$ glacial acetic acid, $\mathrm{pH}$ 4.1-4.3) for 15 minutes followed by ELF97 substrate (Life Tech, catalog E6589) diluted at 1:40 for 5 minutes. The ELF97 substrate generates a yellow fluorescence signal when cleaved by TRAP. For dynamic histomorphometry, mice received calcein (15 $\mathrm{mg} / \mathrm{kg}$, MilliporeSigma) at 9 days (for adult mice) or 4 days (for adolescent mice) before euthanization and xylenol orange $(90 \mathrm{mg} / \mathrm{kg}$, MilliporeSigma) at 2 days before euthanization. Sagittal cryosections of tibiae prepared with cryofilm tape were used for dynamic histomorphometry. Sections were scanned by a Nikon Eclipse 90i fluorescence microscope and areas within secondary spongiosa were quantified by Bioquant Osteo Software (Bioquant Image Analysis). The primary indices include total tissue area (TV), trabecular bone perimeter (BS), single- and double-labeled surface, and interlabel width. Mineralizing surface (MS), mineral apposition rate (MAR, $\mu \mathrm{m} / \mathrm{d}$ ), and surfacereferent bone formation rate (BFR/BS, $\mu \mathrm{m}^{3} / \mu \mathrm{m}^{2} / \mathrm{d}$ ) were calculated as described by Dempster et al. (63).

Cell culture. For CFU-F assay, flushed bone marrow cells were plated at $3 \times 10^{6}$ cells/T25 flask. Cells were cultured in growth medium ( $\alpha$-MEM supplemented with 15\% FBS, 0.1\% $\beta$-mercaptoethanol, $20 \mathrm{mM}$ glutamine, $100 \mathrm{IU} / \mathrm{mL}$ penicillin, and $100 \mu \mathrm{g} / \mathrm{mL}$ streptomycin) for 7 days before counting CFU-F number.

Mesenchymal progenitors were obtained by culturing bone marrow cells at a high density $\left(30 \times 10^{6}\right.$ cells/T25 flask). Once confluent, cells were switched to either adipogenic medium (DMEM with $10 \%$ FBS, $10 \mathrm{ng} / \mathrm{mL}$ triiodothyronine, $1 \mu \mathrm{M}$ rosiglitazone, $1 \mu \mathrm{M}$ dexamethasone, $10 \mu \mathrm{g} / \mathrm{mL}$ insulin, $100 \mathrm{IU} / \mathrm{mL}$ penicillin, and $100 \mu \mathrm{g} / \mathrm{mL}$ streptomycin) for 7 days followed by Oil Red O staining or osteogenic medium ( $\alpha$ MEM with 10\% FBS, $10 \mathrm{nM}$ dexamethasone, $10 \mathrm{mM}$ $\beta$-glycerophosphate, $50 \mu \mathrm{g} / \mathrm{mL}$ ascorbic acid, $100 \mathrm{IU} / \mathrm{mL}$ penicillin, and $100 \mu \mathrm{g} / \mathrm{mL}$ streptomycin) for 2 weeks followed by alizarin Red staining. Brightfield and fluorescence images of cell culture were taken by fluorescence inverted microscopy (Nikon Eclipse, TE2000-U).

For in vitro osteoclastogenesis, bone marrow cells flushed from long bones were seeded to dishes for obtaining bone marrow macrophages (BMMs) as described previously $(64,65)$. BMMs were then seeded at $2 \times 10^{6}$ cells/well in 24-well plates and stimulated with 100 $\mathrm{ng} / \mathrm{mL}$ RANKL and $20 \mathrm{ng} / \mathrm{mL}$ M-CSF (R\&D Systems) for 5 days to generate mature OCs. TRAP staining was performed using a TRAP kit (MilliporeSigma, catalog 387A). Osteoclasts were quantified by counting the number of TRAP $^{+}$multinucleated cells ( $>3$ nuclei/cell) per well.

Serum biochemistry assay. Sera were collected from $W T$ and RANKL-CKO Adipoq mice during euthanization for measuring bone turnover markers, collagen type I C-telopeptide degradation products (CTX-I RatLaps EIA; Immunodiagnostic Systems) and N-terminal propeptide of type I procollagen (PINP ELISA Kit, MyBioSource) respectively, according to the manufacturer's instructions.

RNA analysis. To quantify the expression level of marker genes, total RNA was collected in Tri Reagent (MilliporeSigma) for RNA puri- 
fication. A TaqMan Reverse Transcription Kit (Applied BioSystems) was used to reverse transcribe mRNA into cDNA. The power SYBR Green PCR Master Mix Kit (Applied BioSystems) was used for qRTPCR. The primer sequences for the genes used in this study are listed in Supplemental Table 4.

Statistics. Data are expressed as mean \pm SD and analyzed by $t$ test, 1-way ANOVA with Dunnett's or Turkey's posttest, and 2-way ANOVA with Turkey's posttest for multiple comparisons using Prism 8 software (GraphPad Software). For assays using primary cells, experiments were repeated independently at least 3 times and representative data were shown here. $P$ values of less than 0.05 were considered statistically significant.

Study approval. All animal work performed in this report was approved by the IACUC at the University of Pennsylvania.

Data availability. Sequencing data have been deposited in the Gene Expression Omnibus (GEO) database under accession code GSE145477.

\section{Author contributions}

WY and LQ designed the study. WY and YW performed animal experiments. LY helped with library construction. WY, LZ, XJ and
TG performed histology and imaging analysis. WY, ZL, and LZ performed cell culture and qRT-PCR experiments. $\mathrm{NH}$ performed flow analysis on hematopoietic cells. WY, LY, and LQ performed computational analyses. HK and YC generated Tnfsf11flox mice. WT, ND, XSL, SY, YC, and JA provided administrative, technical, or material support and consultation. LQ wrote the manuscript. WY, LZ, LY, ND, SY, YC, and JA reviewed and revised the manuscript. LQ approved the final version.

\section{Acknowledgments}

This study was supported by NIH grants NIH/NIAMS R01AR066098, R21AR074570 (to LQ), R00AR067283 (to ND), R01AR 066101 (to SY), AR0569546 (to YC), and P30AR069619 (to Penn Center for Musculoskeletal Disorders).

Address correspondence to: Ling Qin, Department of Orthopaedic Surgery, Perelman School of Medicine, University of Pennsylvania, 311A Stemmler Hall, 36th Street and Hamilton Walk, Philadelphia, Pennsylvania 19104, USA. Phone: 215.898.6697, Email: qinling@pennmedicine.upenn.edu.
1. Kains, JA on behalf of the World Health Organisation Scientific Group. Assessment of osteoporosis at the primary health care level. WHO Collaborating Centre for Metabolic Bone Diseases, University of Sheffield 2007. https://www.sheffield. ac.uk/FRAX/pdfs/WHO_Technical_Report.pdf.

2. Ono T, Nakashima T. Recent advances in osteoclast biology. Histochem Cell Biol. 2018;149(4):325-341.

3. Boyle WJ, Simonet WS, Lacey DL. Osteoclast differentiation and activation. Nature. 2003;423(6937):337-342.

4. Kim N, Odgren PR, Kim DK, Marks SC, Choi $\mathrm{Y}$. Diverse roles of the tumor necrosis factor family member TRANCE in skeletal physiology revealed by TRANCE deficiency and partial rescue by a lymphocyte-expressed TRANCE transgene. Proc Natl Acad Sci U S A. 2000;97(20):10905-10910.

5. Kong YY, et al. OPGL is a key regulator of osteoclastogenesis, lymphocyte development and lymph-node organogenesis. Nature. 1999;397(6717):315-323.

6. Suda T, Takahashi N, Udagawa N, Jimi E, Gillespie MT, Martin TJ. Modulation of osteoclast differentiation and function by the new members of the tumor necrosis factor receptor and ligand families. Endocr Rev. 1999;20(3):345-357.

7. Corral DA, et al. Dissociation between bone resorption and bone formation in osteopenic transgenic mice. Proc Natl Acad Sci U S A. 1998;95(23):13835-13840.

8. Ogata N, Kawaguchi H, Chung UI, Roth SI, Segre GV. Continuous activation of G alpha $\mathrm{q}$ in osteoblasts results in osteopenia through impaired osteoblast differentiation. J Biol Chem. 2007;282(49):35757-35764.

9. Xiong J, Onal M, Jilka RL, Weinstein RS, Manolagas SC, O'Brien CA. Matrix-embedded cells control osteoclast formation. Nat Med. 2011;17(10):1235-1241.

10. Nakashima T, et al. Evidence for osteocyte reg- ulation of bone homeostasis through RANKI expression. Nat Med. 2011;17(10):1231-1234.

11. Xiong J, et al. Osteocytes, not osteoblasts or lining cells, are the main source of the RANKL required for osteoclast formation in remodeling bone. PLoS One. 2015;10(9):e0138189.

12. Zhong L, et al. Single cell transcriptomics identifies a unique adipose lineage cell population that regulates bone marrow environment. Elife. 2020;9:e54695.

13. Liu J, Xu Z, Wu W, Wang Y, Shan T. Cre Recombinase strains used for the study of adipose tissues and adipocyte progenitors. JCell Physiol. 2017;232(10):2698-2703.

14. Chandra A, et al. Suppression of sclerostin alleviates radiation-induced bone loss by protecting bone-forming cells and their progenitors through distinct mechanisms. J Bone Miner Res. 2017;32(2):360-372.

15. Ono N, Ono W, Nagasawa T, Kronenberg HM. A subset of chondrogenic cells provides early mesenchymal progenitors in growing bones. Nat Cell Biol. 2014;16(12):1157-1167.

16. Baryawno N, et al. A cellular taxonomy of the bone marrow stroma in homeostasis and leukemia. Cell. 2019;177(7):1915-1932.e16.

17. Tikhonova AN, et al. The bone marrow microenvironment at single-cell resolution. Nature. 2019;569(7755):222-228.

18. Matsushita Y, et al. A Wnt-mediated transformation of the bone marrow stromal cell identity orchestrates skeletal regeneration. Nat Commun. 2020;11(1):332.

19. Wolock SL, et al. Mapping distinct bone marrow niche populations and their differentiation paths. Cell Rep. 2019;28(2):302-311.e5.

20. Kim JH, Kim N. Regulation of NFATc1 in osteoclast differentiation. J Bone Metab. 2014;21(4):233-241.

21. Ishii KA, et al. Coordination of PGC-1beta and iron uptake in mitochondrial biogenesis and osteoclast activation. Nat Med. 2009;15(3):259-266.
22. Kim JH, et al. The mechanism of osteoclast differentiation induced by IL-1. J Immunol. 2009;183(3):1862-1870.

23. Adamik J, et al. EZH2 supports osteoclast differentiation and bone resorption via epigenetic and cytoplasmic targets. JBone Miner Res. 2020;35(1):181-195.

24. Yamoah K, et al. High-mobility group box proteins modulate tumor necrosis factor-alpha expression in osteoclastogenesis via a novel deoxyribonucleic acid sequence. Mol Endocrinol. 2008;22(5):1141-1153.

25. Gautier EL, et al. Systemic analysis of PPAR $\gamma$ in mouse macrophage populations reveals marked diversity in expression with critical roles in resolution of inflammation and airway immunity. Jimmunol. 2012;189(5):2614-2624.

26. Heath V, et al. C/EBPalpha deficiency results in hyperproliferation of hematopoietic progenitor cells and disrupts macrophage development in vitro and in vivo. Blood. 2004;104(6):1639-1647.

27. Huber R, Pietsch D, Panterodt T, Brand K. Regulation of $\mathrm{C} / \mathrm{EBP} \beta$ and resulting functions in cells of the monocytic lineage. Cell Signal. 2012;24(6):1287-1296.

28. Palaga $T$, et al. Notch signaling is activated by TLR stimulation and regulates macrophage functions. Eur JImmunol. 2008;38(1):174-183.

29. Kalajzic I, et al. Use of type I collagen green fluorescent protein transgenes to identify subpopulations of cells at different stages of the osteoblast lineage. JBone Miner Res. 2002;17(1):15-25.

30. Roato I, et al. IL-7 up-regulates TNF-alphadependent osteoclastogenesis in patients affected by solid tumor. PLoS One. 2006;1:e124.

31. Chen Z, Buki K, Vääräniemi J, Gu G, Väänänen HK. The critical role of IL-34 in osteoclastogenesis. PLoS One. 2011;6(4):e18689.

32. Kim MS, Day CJ, Selinger CI, Magno CL, Stephens SR, Morrison NA. MCP-1-induced human osteoclast-like cells are tartrate-resistant acid phosphatase, NFATc1, and calcitonin recep- 
tor-positive but require receptor activator of NFkappaB ligand for bone resorption. J Biol Chem. 2006;281(2):1274-1285.

33. Lu X, et al. VCAM-1 promotes osteolytic expansion of indolent bone micrometastasis of breast cancer by engaging $\alpha 4 \beta 1$-positive osteoclast progenitors. Cancer Cell. 2011;20(6):701-714.

34. Tu Z, Bu H, Dennis JE, Lin F. Efficient osteoclast differentiation requires local complement activation. Blood. 2010;116(22):4456-4463.

35. Honma M, et al. RANKL subcellular trafficking and regulatory mechanisms in osteocytes. JBone Miner Res. 2013;28(9):1936-1949.

36. Xiong J, et al. Soluble RANKL contributes to osteoclast formation in adult mice but not ovariectomy-induced bone loss. Nat Commun. 2018;9(1):2909.

37. Goto $\mathrm{H}$, et al. Human bone marrow adipocytes support dexamethasone-induced osteoclast differentiation and function through RANKL expression. Biomed Res. 2011;32(1):37-44.

38. Goto H, et al. Primary human bone marrow adipocytes support TNF- $\alpha$-induced osteoclast differentiation and function through RANKL expression. Cytokine. 2011;56(3):662-668.

39. Takeshita S, Fumoto T, Naoe Y, Ikeda K. Age-related marrow adipogenesis is linked to increased expression of RANKL. J Biol Chem. 2014;289(24):16699-16710.

40. Holt V, Caplan AI, Haynesworth SE. Identification of a subpopulation of marrow MSC-derived medullary adipocytes that express osteoclast-regulating molecules: marrow adipocytes express osteoclast mediators. PLoS One. 2014;9(10):e108920.

41. Hardouin P, Rharass T, Lucas S. Bone marrow adipose tissue: to be or not to be a typical adipose tissue? Front Endocrinol (Lausanne). 2016;7:85.

42. Fan Y, et al. Parathyroid hormone directs bone marrow mesenchymal cell fate. Cell Metab. 2017;25(3):661-672.

43. Ikebuchi $Y$, et al. Coupling of bone resorption and formation by RANKL reverse signalling. Nature. 2018;561(7722):195-200.
44. Zhao C, et al. Bidirectional ephrinB2-EphB4 signaling controls bone homeostasis. Cell Metab. 2006;4(2):111-121.

45. Mukohira H, et al. Mesenchymal stromal cells in bone marrow express adiponectin and are efficiently targeted by an adiponectin promoter-driven Cre transgene. Int Immunol. 2019;31(11):729-742.

46. Fujiwara Y, Piemontese M, Liu Y, Thostenson JD, Xiong J, O'Brien CA. RANKL (receptor activator of NFKB ligand) produced by osteocytes is required for the increase in b cells and bone loss caused by estrogen deficiency in mice. J Biol Chem. 2016;291(48):24838-24850.

47. Onal M, et al. Receptor activator of nuclear factor $\kappa B$ ligand (RANKL) protein expression by $\mathrm{B}$ lymphocytes contributes to ovariectomy-induced bone loss. J Biol Chem. 2012;287(35):29851-29860.

48. Ginhoux F, Guilliams M. Tissue-resident macrophage ontogeny and homeostasis. Immunity. 2016;44(3):439-449.

49. Jacome-Galarza CE, et al. Developmental origin, functional maintenance and genetic rescue of osteoclasts. Nature. 2019;568(7753):541-545.

50. Yahara Y, et al. Erythromyeloid progenitors give rise to a population of osteoclasts that contribute to bone homeostasis and repair. Nat Cell Biol. 2020;22(1):49-59.

51. Heideveld E, van den Akker E. Digesting the role of bone marrow macrophages on hematopoiesis. Immunobiology. 2017;222(6):814-822.

52. Qin L, Raggatt LJ, Partridge NC. Parathyroid hormone: a double-edged sword for bone metabolism. Trends Endocrinol Metab. 2004;15(2):60-65

53. Khosla S, Hofbauer LC. Osteoporosis treatment: recent developments and ongoing challenges. Lancet Diabetes Endocrinol. 2017;5(11):898-907.

54. Ovchinnikov DA, Deng JM, Ogunrinu G, Behringer RR. Col2a1-directed expression of Cre recombinase in differentiating chondrocytes in transgenic mice. Genesis. 2000;26(2):145-146.

55. Eguchi J, et al. Transcriptional control of adipose lipid handling by IRF4. Cell Metab.
2011;13(3):249-259.

56. Lu Y, Xie Y, Zhang S, Dusevich V, Bonewald LF, Feng JQ. DMP1-targeted Cre expression in odontoblasts and osteocytes. J Dent Res. 2007;86(4):320-325.

57. Stuart $\mathrm{T}$, et al. Comprehensive integration of single-cell data. Cell. 2019;177(7):1888-1902.e21.

58. Huang da W, Sherman BT, Lempicki RA. Systematic and integrative analysis of large gene lists using DAVID bioinformatics resources. Nat Protoc. 2009;4(1):44-57.

59. Kanamori M, Konno H, Osato N, Kawai J, Hayashizaki Y, Suzuki H. A genome-wide and nonredundant mouse transcription factor database. Biochem Biophys Res Commun. 2004;322(3):787-793.

60. Efremova M, Vento-Tormo M, Teichmann SA, Vento-Tormo R. CellPhoneDB: inferring cell-cell communication from combined expression of multi-subunit ligand-receptor complexes. Nat Protoc. 2020;15(4):1484-1506.

61. Bouxsein ML, Boyd SK, Christiansen BA, Guldberg RE, Jepsen KJ, Müller R. Guidelines for assessment of bone microstructure in rodents using micro-computed tomography. J Bone Miner Res. 2010;25(7):1468-1486.

62. Dyment NA, et al. Gdf5 progenitors give rise to fibrocartilage cells that mineralize via hedgehog signaling to form the zonal enthesis. Dev Biol. 2015;405(1):96-107.

63. Dempster DW, et al. Standardized nomenclature, symbols, and units for bone histomorphometry: a 2012 update of the report of the ASBMR Histomorphometry Nomenclature Committee. J Bone Miner Res. 2013;28(1):2-17.

64. Ng AYH, et al. Regulator of $\mathrm{G}$ protein signaling 12 enhances osteoclastogenesis by suppressing Nrf2-dependent antioxidant proteins to promote the generation of reactive oxygen species. Elife. 2019;8:e42951.

65. Yuan X, et al. Regulators of $G$ protein signaling 12 promotes osteoclastogenesis in bone remodeling and pathological bone loss. Cell Death Differ. 2015;22(12):2046-2057. 